Pure and Applied Mathematics Quarterly

Volume 1, Number 4

(Special Issue: In Memory of

Armand Borel, Part 3 of 3 )

$701-735,2005$

\title{
Average Values of Modular $L$-series Via the Relative Trace Formula
}

Dinakar Ramakrishnan and Jonathan Rogawski

In memory of Armand Borel

\section{Contents}

1. Introduction 702

2. The geometric side: Isolation of the main term 704

2.1. Regularization and the main integral 704

2.2. Coset representatives and centralizers 706

2.3. The test function 707

2.4. The dominant terms 708

2.5. The remaining singular terms 714

$\begin{array}{ll}\text { 2.6. The regular terms } & 715\end{array}$

3. A bound for the sum of regular terms 723

4. The spectral side $\quad 725$

5. Proof of the Main Theorem 728

$\begin{array}{lll}\text { 6. } & \text { The measure } & 728\end{array}$

$\begin{array}{ll}\text { Bibliography } & 734\end{array}$

Received August 17, 2005. 


\section{Introduction}

Let $k>2$ be an even integer and $N$ a prime. Let $\mathcal{S}_{N}(k)$ be the space of holomorphic cuspforms $\varphi$ of weight $k$ for the congruence subgroup $\Gamma_{0}(N)$, acting on the upper half plane $\mathcal{H}$. For $\varphi \in S_{N}(k)$, we write $(\varphi, \varphi)$ for the Petersson norm and $L(s, \varphi)$ for the L-function, normalized so that the functional equation relates $s$ and $1-s$. Let $\mathcal{F}_{N}(k)^{\text {new }}$ be an orthogonal basis of Hecke-eigenforms spanning the subspace new forms.

In addition to $k$ and $N$, we fix the following data throughout the paper: a primitive quadratic character $\chi$ of conductor $D<0$ such that $(N, D)=1$ and $\chi(-N)=1$ and a prime $p$ such that $(p, D N)=1$. Let $L(s, \chi)$ be the Dirichlet $L$-function $\sum_{n \geq 1} \chi(n) n^{-s}$.

The Sato-Tate measure is the measure on the interval $[-2,2]$ defined by

$$
\mu_{\infty}=\frac{1}{2 \pi} \sqrt{4-x^{2}} d x
$$

We define two probability measures $\mu_{+}(x) d x$ and $\mu_{-}(x) d x$ on $[-2,2]$ by

$$
\begin{aligned}
& \mu_{+}=\frac{p-1}{\left(p^{1 / 2}+p^{-1 / 2}-x\right)^{2}} \mu_{\infty} \\
& \mu_{-}=\frac{p+1}{\left(p^{1 / 2}+p^{-1 / 2}\right)^{2}-x^{2}} \mu_{\infty}
\end{aligned}
$$

Let $\mu_{p}$ be $\mu_{+}$or $\mu_{-}$according as $\chi(p)$ is 1 or -1 .

Let $d_{k}$ be the formal degree of the holomorphic discrete series representation $\mathcal{D}_{k}$ of $\operatorname{PGL}(2, \mathbb{R})$ of weight $k$, defined relative to the Haar measure $d g_{\infty}$ on $\operatorname{GL}(2, \mathbb{R})$ whose push-forward to $\mathcal{H}$ is the measure used to define the Petersson norm $(\varphi, \varphi)$. For the standard measure $y^{-2} d x d y, d_{k}=(k-1) / 2$. We define the following constant

$$
c_{k}=2^{k} d_{k} e_{k} \frac{k\left[\left(\frac{k}{2}-1\right) !\right]^{2}}{(k-1) !}
$$

where

$$
e_{k}=1+\sum_{n=0}^{\frac{k}{2}-2}\left(\begin{array}{c}
k \\
2 n+1
\end{array}\right)(-1)^{\frac{k}{2}-n}\left(\frac{k}{2}+n-1\right) !\left(\frac{k}{2}-n-2\right) !
$$

For $\varphi \in \mathcal{F}_{N}(k)^{\text {new }}$, let $a_{p}(\varphi)$ denotes the normalized $p$-Hecke eigenvalue of $\varphi$, which is known by Deligne to lie in $[-2,2]$. If $c_{p}$ is the usual $p$-Hecke eigenvalue, which is an algebraic integer, then $a_{p}=c_{p} / p^{(k-1) / 2}$. Our main result is the following: 
Theorem A Let $k>2$ be an even integer, $\chi=\chi_{D}$ a quadratic character of conductor $D<0, N$ a prime with $\chi(-N)=1$, and $p$ a prime not dividing $N D$. Then for any subinterval $J$ of $[-2,2]$, we have $(\forall \varepsilon>0)$ :

$$
\sum_{\substack{\varphi \in \mathcal{F}_{N}(k)^{\text {new }} \\ a_{p}(\varphi) \in J}} \frac{L\left(\frac{1}{2}, \varphi \otimes \chi\right) L\left(\frac{1}{2}, \varphi\right)}{(\varphi, \varphi)}=2 c_{k} L(1, \chi) \mu_{p}(J)+O\left(N^{-k / 2+\varepsilon}\right)
$$

Corollary B Let $k>2$ be an even integer, $\chi$ a quadratic character of conductor $D<0$, and $p$ a prime such that $(p, D)=1$. Let $J \subset[-2,2]$ be a non-empty interval. Then for all sufficiently large primes $N$ not dividing $p D$, which are inert in $K=\mathbb{Q}[\sqrt{D}]$, there exists a holomorphic newform $\varphi$ of weight $k$ for $\Gamma_{0}(N)$ such that:

(i) $a_{p}(\varphi) \in J$; and

(ii) $L(1 / 2, \varphi \otimes \chi) L(1 / 2, \varphi) \neq 0$.

In particular, there are cusp forms $\varphi$ of weight $k$ and prime level for which $a_{p}$ lies arbitrarily close to 2 or -2 , and $L(1 / 2, \varphi \otimes \chi) L(1 / 2, \varphi)$ is non-zero. Without the requirement on the non-vanishing of the $L$-value, this is a well known result of Serre ([Se]); see also Sarnak ([Sa]) in the context of Maass forms.

Following a suggestion of Philippe Michel, we observe that the measure $\mu_{p}$ can be written in the following form. For $x \in[-2,2]$, let $\pi_{x}$ be the unramified representation of $P G L_{2}\left(\mathbf{Q}_{p}\right)$ whose Satake parameters are $\left\{p^{s}, p^{-s}\right\}$ where $x=$ $p^{s}+p^{-s}$. The local factors $L\left(s, \pi_{x}\right)$ and $L\left(\frac{1}{2}, \pi_{x} \otimes \chi_{p}\right)$ are then defined and we have:

$$
\mu_{p}=\frac{L\left(\frac{1}{2}, \pi_{x}\right) L\left(\frac{1}{2}, \pi_{x} \otimes \chi_{p}\right)}{L(1, \chi)} \mu_{\infty}
$$

When $\chi(p)=-1, \mu_{p}=\mu_{-}$is the familiar spherical Plancherel measure for $P G L_{2}\left(\mathbf{Q}_{p}\right)$. In either case, $\mu_{p}$ approaches $\mu_{\infty}$ as $p$ tends to $\infty$. Theorem A can be reformulated in terms of the product

$$
L^{p}\left(\frac{1}{2}, \varphi \otimes \chi\right) L^{p}\left(\frac{1}{2}, \varphi\right)
$$

where $L^{p}$ is the L-function with the $p$-Euler factor is removed. In this case, the measure $\mu_{p}$ is replaced by $\mu_{\infty} / L(1, \chi)$. We note that, unlike $\mu_{-}$and $\mu_{\infty}$, the measure $\mu_{+}$has a bias favoring positive $x$.

When $J$ is the full interval $[-2,2]$, the measure $\mu_{p}$ is not needed and the result of Theorem A has then been known for some time (for $k=2$ ) by the work of W. Duke ([Du]); see also [Lu]. Our work began with our effort to understand these papers. It should be mentioned that in $([\mathrm{IwS}])$, Iwaniec and Sarnak introduce 
a novel program to prove a good lower bound for $L(1, \chi)$ from a simultaneous lower bound, for a family of $\varphi$, of $L\left(\frac{1}{2}, \varphi\right)$ and $L\left(\frac{1}{2}, \varphi \otimes \chi\right)$. There are other notable papers dealing with non-vanishing results for modular $L$-functions and their derivatives; see for example [KMV].

Our approach makes use of the relative trace formula of $\mathrm{H}$. Jacquet based on the integral of the GL(2) kernel over the square of the maximal split torus. We apply the trace formula to a certain factorizable function $f$ on $\operatorname{GL}(2, \mathbb{A})$ where $f_{\infty}$ is a matrix coefficient of $\mathcal{D}_{k}$. Since the approach taken here requires that $f_{\infty}$ be integrable, we are forced to assume that $k>2$. With additional work, the method can be carried out for $k=2$.

Much of the work on this paper was done five years ago, but the explicit nature of the measure was worked out only recently. One can extend the result without much trouble to square-free level $N$ with $\chi(-N)=1$. However, if $\chi(-N)=-1$, the relevant $L$-function vanishes at $s=1 / 2$ and in that case one should consider the derivative. Our method should also be applicable to analogous situations over totally real fields, even with varying weights $\geq 2$ at the different infinite places, but of the same parity. Similarly, one should be able to treat the case of Maass forms, but then the infinity type $\lambda$ will not be fixed, but will lie in a short interval. We thank Nathaniel Grossman for helpful comments on the evaluation of an archimedean integral involving the hypergeometric function. The first author also thanks Bill Duke, Jeff Hoffstein, Hervé Jacquet, Wenzhi Luo, Philippe Michel, and S. Rallis for interesting conversations. After this paper was finalized, we heard from P. Michel of a similar work of E. Royer [Ro] involving the average of the single $L$-function $L(1 / 2, \varphi)$, without using representation theory. Last but not least, we acknowledge with thanks the support from the National Science Foundation.

\section{Relative Trace Formula: the geometric side}

2.1. Regularization of the Relative Trace Formula. Let $G$ denote $\mathrm{GL}(2) / \mathbb{Q}$ with center $Z$, and set $\widetilde{G}=G / Z$. Consider the kernel

$$
K(x, y)=\sum_{\gamma \in \widetilde{G}(\mathbb{Q})} f\left(x^{-1} \gamma y\right)
$$

where $f$ is a suitable factorizable, smooth function on $\widetilde{G}(\mathbb{A}$ ) (specified in $\S 2.2$ below). Let $T$ denote diagonal subgroup of $G$ and set $\widetilde{T}=T / Z$. We make use of a relative trace formula due to Jacquet ([Ja]) which involves integration of this kernel over the square of $\widetilde{T}(\mathbb{A}) / \widetilde{T}(\mathbb{Q})$ against a character. Since the integral is not absolutely convergent. However, rather than truncate the kernel, we shall regularize the integral by computing it as a limit. 
Let $\chi$ be a non-trivial, quadratic character of $\mathbb{A}^{*}$ where $\mathbb{A}$ is the ring of adeles of $\mathbb{Q}$, and $\mathbb{A}^{*}$ is the multiplicative group of ideles. For $s_{1}, s_{2} \in \mathbb{C}$ and $c>0$, define

$$
I_{c}\left(f ; s_{1}, s_{2}\right)=\iint_{\mathbb{Q}^{*} \backslash \mathbb{A}^{*} \times \mathbb{Q}^{*} \backslash \mathbb{A}^{*}}^{c} K\left(\left(\begin{array}{ll}
a & 0 \\
0 & 1
\end{array}\right),\left(\begin{array}{ll}
b & 0 \\
0 & 1
\end{array}\right)\right) \chi(a)|a|^{s_{1}}|b|^{s_{2}} d^{*} a d^{*} b
$$

where the superscript $c$ indicates that the integrals are taken over $a, b \in \mathbb{Q}^{*} \backslash \mathbb{A}^{*}$ such that $c^{-1}<|a|,|b|<c$. We use the multiplicative Haar measure on $\mathbb{A}^{*}$ which is a product of the measures $d x /|x|$ on $\mathbb{R}^{*}$ (where $d x$ is Lebesgue measure on $\mathbb{R}$ ) and the measures on $\mathbb{Q}_{v}^{*}$, for finite $v$, assigning measure one to $\mathbb{Z}_{v}^{*}$.

We will see in $\S 4$ that the following limit exists for all $s_{1}$ and $s_{2}$ :

$$
I\left(f ; s_{1}, s_{2}\right):=\lim _{c \rightarrow \infty} I_{c}\left(f ; s_{1}, s_{2}\right) .
$$

Our main object of study is the value at $s_{1}=s_{2}=0$ :

$$
I(f):=I(f ; 0,0)
$$

2.2. The test function. Let $S^{\prime}$ be the set of finite primes $q$ at which $\chi$ is ramified. Fix two distinct primes $p, N \notin S^{\prime}$ and set

$$
S=S^{\prime} \cup\{p, N, \infty\}
$$

We define a function $f$ on $\widetilde{G}(\mathbb{A})$ of the form

$$
f=f_{\infty} \times f_{p} \times f_{N} \times f_{S^{\prime}} \times f^{S}
$$

where $f_{S^{\prime}}=\prod_{v \in S^{\prime}} f_{v}$ and $f^{S}=\prod_{v \notin S} f_{v}$.

For any prime $v$, a subscript $v$ denotes localization at $v$. For example, $F_{v}=$ $F\left(\mathbb{Q}_{v}\right), Z_{v}=Z\left(\mathbb{Q}_{v}\right)$, etc. As in the introduction, let $\mathcal{D}_{k}$ be the holomorphic discrete series representation of $\widetilde{G}_{v}=\operatorname{PGL}(2, \mathbb{R})$ of weight $k$ and let $d_{k}$ be its formal degree. We choose the archimedean function $f_{\infty}$ to be $d_{k}$ times the complex conjugate of the matrix coefficient $\left\langle\mathcal{D}_{k}(g) v, v\right\rangle$ where $v$ is a unit vector of lowest weight $k$. Explicitly:

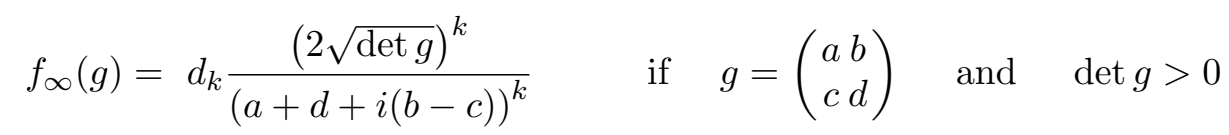

and $f_{\infty}(g)=0$ if $\operatorname{det} g<0$.

Put $K_{\infty}=S O(2)$ and for $v=p$ set $K_{v}=\mathrm{GL}\left(2, \mathbb{Z}_{p}\right)$. Let $\widetilde{K}_{v}$ the the subgroup $Z_{v} \backslash Z_{v} K_{v}$ of $\widetilde{G}_{v}$. Let $\mathcal{H}_{p}$ be the algebra of compactly supported, bi- $\widetilde{K}_{v}$-invariant functions of $\widetilde{G}\left(\mathbb{Q}_{p}\right)$. We take the function $f_{p}$ to be an arbitrary element of $\mathcal{H}_{p}$. Let

$$
K_{0}(N)=\left\{\left(\begin{array}{l}
a b \\
c d
\end{array}\right) \in G L_{2}\left(\mathbf{Z}_{N}\right): c \equiv 0(\bmod N)\right\} .
$$


and let $\widetilde{K}_{0}(N)$ be the subgroup $Z_{N} \backslash Z_{N} K_{0}(N)$ of $\widetilde{G}_{N}$. Let $V_{N}$ be the measure of $\widetilde{K}_{0}(N)$. Then we take $f_{N}$ to be the characteristic function of $\widetilde{K}_{0}(N)$ divided by $V_{N}$.

We let $f^{S}$ be the characteristic function of $\prod_{v \notin S} \widetilde{K}_{v}$.

Finally let $v=q$ be in $S^{\prime}$. If $q^{m}$ is the conductor of $\chi_{q}$, denote by $X$ the set of representatives in $q^{-m} \mathbb{Z}_{q} \subset \mathbb{Q}_{q}$ for $q^{-m} \mathbb{Z}_{q} / \mathbb{Z}_{q}$, which is a finite group isomorphic to $\mathbb{Z} / q^{m}$. We may view $\chi_{v}$ as a character of $X$. Put

$$
f_{v}=g\left(\chi_{v}\right)^{-1} \sum_{z \in X} \chi_{1, v}(z) f_{z, v}
$$

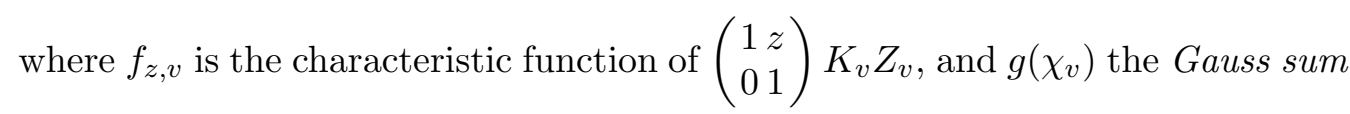

$$
g\left(\chi_{v}\right)=\int_{\mathbb{Z}_{q}^{*}} \chi_{v}(x) \psi_{v}\left(q^{-m} x\right) d^{*} x .
$$

Here $\psi$ denotes the additive character of $\mathbb{Q}_{q}$ defined as the composite

$$
\mathbb{Q}_{q} \rightarrow \mathbb{Q}_{q} / \mathbb{Z}_{q} \rightarrow \mathbb{Q} / \mathbb{Z} \rightarrow S^{1},
$$

with the last arrow on the right being $x \rightarrow e^{2 \pi i x}$. It is well known that $g\left(\chi_{v}\right)$ has absolute value $q^{m / 2}$. The global Gauss sum $g(\chi)$ is a product of local ones and since $\chi$ is odd,

$$
g(\chi)=i|D|^{1 / 2}
$$

2.3. The Geometric Side. Let $T$ be the diagonal subgroup. Set

$$
\widetilde{T}=\left\{t_{a}\right\} \quad \text { where } \quad t_{a}=\left(\begin{array}{ll}
a & 0 \\
0 & 1
\end{array}\right) .
$$

We identify $\widetilde{T}$ with $T / Z \subset G / Z$. For $\delta \in \widetilde{G}$, define the subgroup

$$
C_{\delta}=\left\{\left(t, t^{\prime}\right) \in \widetilde{T} \times \widetilde{T}: t^{-1} \delta t^{\prime}=z \delta \quad \text { for some } \quad z \in Z\right\} .
$$

We break up the sum over $\gamma$ defining $K(x, y)$ into sums over $\widetilde{T}(\mathbb{Q})$-double cosets in the usual way to obtain

$$
I_{c}\left(f ; s_{1}, s_{2}\right)=\sum_{\{\delta\}} I_{c}\left(\delta, f ; s_{1}, s_{2}\right)
$$

where $\{\delta\}$ is a set of representatives for the double cosets $\widetilde{T} \backslash \widetilde{G} / \widetilde{T}$ and

$$
I_{c}\left(\delta, f ; s_{1}, s_{2}\right)=\int_{C_{\delta}(\mathbb{Q}) \backslash(\widetilde{T}(\mathbb{A}) \times \widetilde{T}(\mathbb{A}))}^{c} f\left(t_{a}^{-1} \delta t_{b}\right) \chi(a)|a|^{s_{1}}|b|^{s_{2}} d^{*} a d^{*} b
$$


The superscript $c$ indicates that the integral is taken over $c^{-1}<|a|, \quad|b|<c$. Define the following limit distribution

$$
I\left(\delta, f ; s_{1}, s_{2}\right)=\lim _{c \rightarrow \infty} I_{c}\left(\delta, f ; s_{1}, s_{2}\right)
$$

When this limit exists, we will say that $I\left(\delta, f ; s_{1}, s_{2}\right)$ converges conditionally. Set

$$
\sigma_{1}=\Re\left(s_{1}\right), \quad \sigma_{2}=\Re\left(s_{2}\right)
$$

We will assume from now on that the following conditions hold:

$$
-\frac{k}{2}+1<\sigma_{1}<\frac{k}{2} \quad \text { and } \quad-\frac{k}{2}<\sigma_{2}<\frac{k}{2}-1
$$

Since $k$ is an even integer and $k>2$, these conditions hold in particular if $-1<\sigma_{1}, \sigma_{2}<1$, which is sufficient for our purposes.

Theorem 2.1. Assume that the conditions $\left(^{*}\right)$ hold. Then $I\left(\delta, f ; s_{1}, s_{2}\right)$ converges conditionally for all $\delta$ and

$$
\sum_{\{\delta\}}\left|I\left(\delta, f ; s_{1}, s_{2}\right)\right|<\infty
$$

In particular,

$$
\left.I\left(f ; s_{1}, s_{2}\right)\right)=\sum_{\{\delta\}} I\left(\delta, f ; s_{1}, s_{2}\right)
$$

2.4. Coset representatives and centralizers. We recall some easily verified facts about the double coset space $\widetilde{T} \backslash \widetilde{G} / \widetilde{T}$. Define matrices

$$
\xi(x)=\left(\begin{array}{ll}
1 & x \\
1 & 1
\end{array}\right)
$$

and

$$
n^{+}=\left(\begin{array}{ll}
1 & 1 \\
0 & 1
\end{array}\right), \quad n^{-}=\left(\begin{array}{ll}
1 & 0 \\
1 & 1
\end{array}\right), \quad \varepsilon=\left(\begin{array}{ll}
0 & 1 \\
1 & 0
\end{array}\right)
$$

Further let $e$ denote the identity matrix in $G$.

Lemma 1. The set of matrices

$$
\{\xi(x): x \neq 0,1\} \cup\left\{e, \quad \varepsilon, \quad n^{+}, \quad \varepsilon n^{+}, \quad n^{-}, \quad \varepsilon n^{-}\right\}
$$

is a set of representatives for the double cosets $\widetilde{T} \backslash \widetilde{G} / \widetilde{T}$.

The elements $\xi(x)$ and the orbits they represent will be called regular. The six remaining representatives and their orbits will be called singular.

We have

$$
\left(\begin{array}{ll}
a^{-1} & \\
& 1
\end{array}\right)\left(\begin{array}{ll}
x & y \\
z & w
\end{array}\right)\left(\begin{array}{c}
b \\
1
\end{array}\right)=\left(\begin{array}{cc}
b a^{-1} x a^{-1} y \\
b z & w
\end{array}\right) .
$$


Lemma 2. $C_{\delta}=\{e\}$ if $\delta$ is regular or if $\delta \in\left\{n^{+}, \varepsilon n^{+}, n^{-}, \varepsilon n^{-}\right\}$. On the other hand,

$$
\begin{aligned}
& C_{e}=\left\{\left(t_{a}, t_{a}\right): a \in I_{\mathbb{Q}}\right\} \\
& C_{\varepsilon}=\left\{\left(t_{a}, t_{a}^{-1}\right): a \in I_{\mathbb{Q}}\right\} .
\end{aligned}
$$

\subsection{Vanishing of the terms attached to $e, \epsilon, \varepsilon n^{ \pm}$.}

Lemma 3. As $\chi$ is non-trivial, for all $s_{1}, s_{2}$, and all $c>0$ we have

$$
I_{c}\left(e, f ; s_{1}, s_{2}\right)=I_{c}\left(\varepsilon, f ; s_{1}, s_{2}\right)=0 .
$$

Proof. To treat $\delta=e$, write a typical element of $\widetilde{T} \times \widetilde{T}$ as $\left(t_{x} t_{a}, t_{a}\right)$. Then we may write $I_{c}(e, f)$ as a double integral

$$
\int_{\mathbb{Q}^{*} \backslash \mathbb{A}^{*}, c^{-1}<|a|<c}\left(\int_{x \in \mathbb{A}^{*}, c^{-1}<x a<c} f\left(t_{x}^{-1}\right) \chi(x)|x|^{s_{1}} d^{*} x\right) \chi(a)|a|^{s_{1}+s_{2}} d^{*} a .
$$

Since $\chi$ is non-trivial and of finite order, its restriction to $I_{\mathbb{Q}}^{1}$ is also non-trivial. Consequently its integral over $a \in \mathbb{Q}^{*} \backslash I_{\mathbb{Q}}^{1}$ vanishes, showing that $I_{c}(e, f)=0$.

To treat $\delta=\varepsilon$, write a typical element of $\widetilde{T} \times \widetilde{T}$ as $\left(t_{x} t_{a}, t_{a}^{-1}\right)$. Then we may write $I_{c}(\varepsilon, f)$ as a double integral

$$
\int_{\mathbb{Q}^{*} \backslash \mathbb{A}^{*}, c<|a|<c^{-1}}\left(\int_{x \in \mathbb{A}^{*}, c^{-1}<x a<c} f\left(t_{x}^{-1}\right) \chi(x)|x|^{s_{1}} d^{*} x\right) \chi(a)|a|^{s_{1}-s_{2}} d^{*} a .
$$

Again, the integral of $\chi$ over $a \in \mathbb{Q}^{*} \backslash I_{\mathbb{Q}}^{1}$ vanishes, implying that $\left.I_{c}(\varepsilon, f)\right)=0$.

The vanishing of $I_{c}\left(e, f ; s_{1}, s_{2}\right)$ and $I_{c}\left(\varepsilon, f ; s_{1}, s_{2}\right)$ does not depend on the specific choice of test function $f$. The next proposition shows that the terms attached to $\varepsilon n^{ \pm}$also vanish for our particular choice of $f$.

Proposition 2.1. If $\delta=\varepsilon n^{+}$or $\varepsilon n^{-}$, then $\left.I_{c}\left(\delta, f ; s_{1}, s_{2}\right)\right)=0$ for all $s_{1}, s_{2}$ and $c>0$.

Proof. For $\delta=\varepsilon n^{+}$, we have

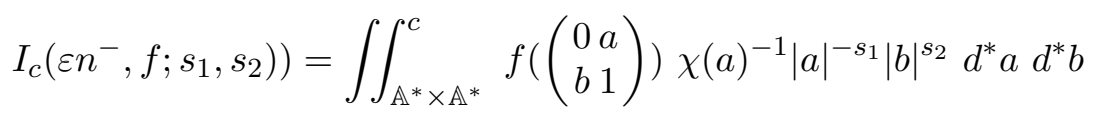

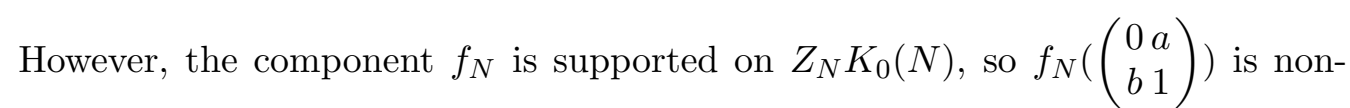
zero for $(a, b) \in\left(\mathbb{Q}_{N}^{*}\right)^{2}$ iff there exists $\lambda \in \mathbb{Q}_{N}^{*}$ such that $\lambda, \lambda a \in \mathbb{Z}_{N}, \lambda b \in$ $N \mathbb{Z}_{N}$ and $\operatorname{det}\left(\lambda\left(\begin{array}{ll}0 & a \\ b & 1\end{array}\right)\right)=-\lambda^{2} a b \in \mathbb{Z}_{N}^{*}$. These conditions cannot be satisfied simultaneously, so $f$ in fact vanishes on the domain of the integral in (1). 
Similarly, for $\delta=\varepsilon n^{-}$, we have

$$
\left.I_{c}\left(\varepsilon n^{-}, f ; s_{1}, s_{2}\right)\right)=\iint_{\mathbb{A}^{*} \times \mathbb{A}^{*}}^{c} f\left(\left(\begin{array}{cc}
a b & a \\
b & 0
\end{array}\right)\right) \chi(a)^{-1}|a|^{-s_{1}}|b|^{s_{2}} d^{*} a d^{*} b
$$

Again, $f\left(\left(\begin{array}{cc}a b & a \\ b & 0\end{array}\right)\right.$ is non-zero only if there exists $\lambda$ in $\mathbb{Q}_{N}^{*}$ such that $\lambda a b, \lambda a \in \mathbb{Z}_{N}$, $\lambda b \in N \mathbb{Z}_{N}$ and $\lambda^{2} a b \in \mathbb{Z}_{N}^{*}$, which is impossible. Thus $\left.I_{c}\left(\varepsilon n^{-}, f ; s_{1}, s_{2}\right)\right)$ also vanishes.

2.6. The dominant terms. We have seen that four of the six singular terms contribute zero to the geometric side of the relative trace formula. It will turn out that since $\chi$ is non-trivial, the terms corresponding to $\delta=n^{ \pm}$are dominant as the level becomes large. Explicitly, these terms are:

$$
\begin{aligned}
& I_{c}\left(n^{+}, f, s_{1}, s_{2}\right)=\iint_{\mathbb{A}^{*} \times \mathbb{A}^{*}}^{c} f\left(\left(\begin{array}{cc}
a b & a \\
0 & 1
\end{array}\right)\right) \chi(a)^{-1}|a|^{-s_{1}}|b|^{s_{2}} d^{*} a d^{*} b \\
& =\iint_{\mathbb{A}^{*} \times \mathbb{A}^{*}}^{c} f\left(\left(\begin{array}{cc}
b & a \\
0 & 1
\end{array}\right)\right) \chi(a)|a|^{-s_{1}-s_{2}}|b|^{s_{2}} d^{*} a d^{*} b
\end{aligned}
$$

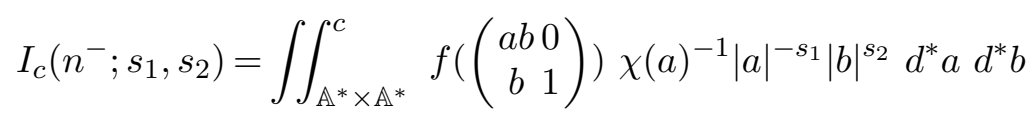

$$
\begin{aligned}
& =\iint_{\mathbb{A}^{*} \times \mathbb{A}^{*}}^{c} f\left(\left(\begin{array}{cc}
b & 0 \\
a & 1
\end{array}\right)\right) \chi\left(a b^{-1}\right)|a|^{s_{1}+s_{2}}|b|^{-s_{1}} d^{*} a d^{*} b .
\end{aligned}
$$

Proposition 2.2. The integrals $I\left(n^{+}, f, s_{1}, s_{2}\right)$ and $I\left(n^{+}, f, s_{1}, s_{2}\right)$ are conditionally convergent and analytic in the region defined by the conditions $\left(^{*}\right)$. Furthermore,

(A) $I\left(n^{+}, f, s_{1}, s_{2}\right)$ is absolutely convergent if $\left(^{*}\right)$ holds and $\sigma_{1}+\sigma_{2}<-1$.

(B) $I\left(n^{-}, f, s_{1}, s_{2}\right)$ is absolutely convergent if $\left(^{*}\right)$ holds and $\sigma_{1}+\sigma_{2}>1$.

To treat the case $\delta=n^{+}$, we define:

$$
g(a)=\int_{\mathbb{A}^{*}} f\left(\left(\begin{array}{ll}
b & a \\
0 & 1
\end{array}\right)\right)|b|^{s_{2}} d^{*} b
$$

Let $\widehat{g}$ be the Fourier transform of $g$ with respect to the additive character $\psi$ fixed above.

Lemma 4. If $-\frac{k}{2}<\sigma_{2}<\frac{k}{2}-1$, then the integral $g(a)$ converges absolutely for all $a \in \mathbb{A}$. Furthermore,
(A) $g(a)$ is $O\left(|a|^{\sigma_{2}-k / 2}\right)$ as $|a| \rightarrow \infty$.
(B) $\hat{g}(a)$ is $O\left(|a|^{-m}\right)$ as $|a| \rightarrow \infty$ for all $m$. 
Proof. The function $f\left(\left(\begin{array}{ll}b & a \\ 0 & 1\end{array}\right)\right)$ is zero unless $a_{f}$ and $b_{f}$ lie in a compact subsets of $\mathbb{A}_{f}$ and $\mathbb{A}_{f}^{*}$, respectively. So is suffices to estimate the archimedean integral:

$$
g_{\infty}(a)=\int_{\mathbb{R}^{*}} f_{\infty}\left(\left(\begin{array}{cc}
b & a \\
0 & 1
\end{array}\right)\right)|b|^{s_{2}} d^{*} b=2^{k} d_{k} \int_{0}^{\infty} \frac{b^{k / 2+s_{2}}}{(b+1-i a)^{k}} d^{*} b
$$

The integral over $[0,1]$ is $O\left(|a|^{-k}\right)$ and

$$
\left|\int_{1}^{\infty} \frac{b^{k / 2+s_{2}}}{(b+1-i a)^{k}} d^{*} b\right|<\int_{1}^{\infty} \frac{b^{k / 2+\sigma_{2}}}{|b-i a|^{k}} d^{*} b<|a|^{s_{2}-\frac{k}{2}} \int_{0}^{\infty} \frac{b^{k / 2+\sigma_{2}}}{|b-i|^{k}} d^{*} b
$$

The integral on the right converges if $\sigma_{2}<\frac{k}{2}$ and Part (A) follows since we assume $\sigma_{2}-\frac{k}{2}>-k$. Furthermore, $g_{\infty}(a)$ is integrable on $\mathbb{R}$ is $\sigma_{2}<\frac{k}{2}-1$ and the same is true of all of its derivatives. Hence $\widehat{g}_{\infty}(a)$ decreases faster than $|a|^{m}$ for all $m$.

Now recall that if $F(a)$ is any smooth function such that $|F(a)|<<|a|^{-\lambda}$ and $|\hat{F}(a)|<<|a|^{-\mu}$, then for any non-trivial unitary Hecke character $\chi$, the following limit exists and is analytic for $1-\mu<\Re(s)<\lambda$ :

$$
\lim _{c \rightarrow \infty} \int_{\mathbb{A}^{*}}^{c} F(a) \chi(a)|a|^{s} d^{*} a
$$

This follows from the Poisson summation formula as in Tate's thesis. We apply this remark to $g(a)$ with $s=-s_{1}-s_{2}$ where $-\frac{k}{2}<\sigma_{2}<\frac{k}{2}-1$. Then, by Lemma 4, may take $\lambda=\frac{k}{2}-\sigma_{2}$ and $\mu$ arbitrary. Hence $I\left(n^{+}, f, s_{1}, s_{2}\right)$ is conditionally convergent if $-\sigma_{1}-\sigma_{2}<\frac{k}{2}-\sigma_{2}$, that is, if $\sigma_{1}>-\frac{k}{2}$. The same considerations apply to of $I\left(n^{-}, f, s_{1}, s_{2}\right)$. The convergence conditions are the same, but with $s_{1}$ and $s_{2}$ replaced by $-s_{2}$ and $-s_{1}$ repectively. The conditions become:

$$
-\frac{k}{2}<-\sigma_{1}<\frac{k}{2}-1 \quad-\sigma_{2}>-\frac{k}{2}
$$

This proves the assertion in Proposition 2.2 about conditional convergence. From the integral above we find that $I\left(n^{+}, f, s_{1}, s_{2}\right)$ (resp. $\left.I\left(n^{-}, f, s_{1}, s_{2}\right)\right)$ converges absolutely if the conditions of conditional convergence are satisfied and $\sigma_{1}+\sigma_{2}<$ -1 (resp. $\sigma_{1}+\sigma_{2}>1$ ). The completes the proof of Proposition 2.2.

We define the local integrals 


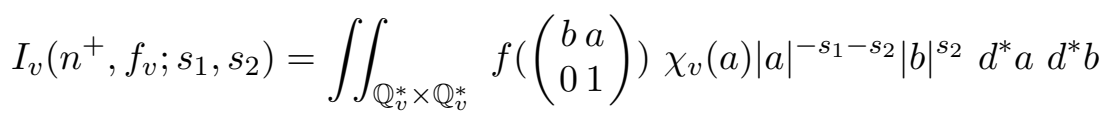

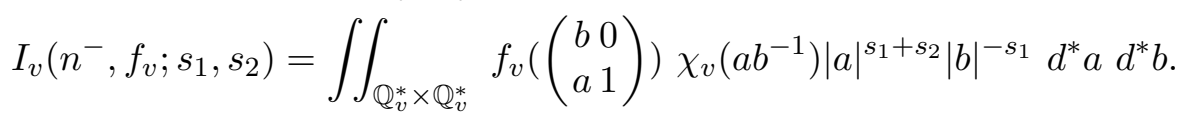

and we set:

$$
\begin{aligned}
& F_{v}\left(n^{+}, f_{v} ; s_{1}, s_{2}\right)=\frac{I_{v}\left(n^{+}, f_{v} ; s_{1}, s_{2}\right)}{L\left(-s_{1}-s_{2}, \chi_{v}\right)} \\
& F_{v}\left(n^{-}, f_{v} ; s_{1}, s_{2}\right)=\frac{I_{v}\left(n^{-}, f_{v} ; s_{1}, s_{2}\right)}{L\left(s_{1}+s_{2}, \chi_{v}\right)}
\end{aligned}
$$

Proposition 2.3. For all $v, F_{v}\left(n^{+}, f_{v} ; s_{1}, s_{2}\right)$ and $F_{v}\left(n^{+}, f_{v} ; s_{1}, s_{2}\right)$ are analytic in the region defined by the conditions $\left(^{*}\right)$ and in particular, at $s_{1}=s_{2}=0$. Let $F_{v}\left(n^{ \pm}, f_{v}\right)=F_{v}\left(n^{ \pm}, f_{v} ; 0,0\right)$. Then:

(a) If $v \notin\{p, N, \infty\}$, then

$$
F_{v}\left(n^{+}, f_{v}\right)=F_{v}\left(n^{-}, f_{v}\right)=g\left(\chi_{v}\right)^{-1}
$$

Note that $g\left(\chi_{v}\right)=1$ if $\chi_{v}$ is unramified.

(b) At $v=N$, we have $F_{v}\left(n^{+}, f_{v}\right)=V_{N}^{-1}$ and $F_{v}\left(n^{-}, f_{v}\right)=\chi_{N}(N) V_{N}^{-1}$

(c) At the archimedean place,

$$
F_{\infty}\left(n^{+}, f_{\infty}\right)=-F_{\infty}\left(n^{-}, f_{\infty}\right)=i \pi c_{k},
$$

where $c_{k}$ is the constant defined in the introduction.

Proof. (a) First let $v=q$ be outside $S=S^{\prime} \cup\{p, N, \infty\}$, corresponding to a prime $q \neq p, N$. By our choice of $f, f_{v}$ is the characteristic function of $Z_{v} K_{v}$. Consequently,

$$
(a, b) \rightarrow f_{v}\left(\begin{array}{cc}
b & a \\
0 & 1
\end{array}\right)
$$

is simply the characteristic function of $\mathbf{Z}_{v} \times \mathbf{Z}_{v}^{*}$. Since $\chi$ is unramified at $v$,

$$
I_{v}\left(n^{+}, f_{v} ; s_{1}, s_{2}\right)=\sum_{n \geq 0} \chi\left(q^{-n}\right) q^{n\left(s_{1}+s_{2}\right)}=L_{v}\left(-s_{1}-s_{2}, \chi_{v}\right)
$$

Hence $F_{v}\left(n^{+}, f_{v} ; s_{1}, s_{2}\right)=1$ for all $s_{1}, s_{1}$, (a) follows in this case. A similar calculation shows that $I_{v}\left(n^{-}, f_{v} ; s_{1}, s_{2}\right)=L_{v}\left(s_{1}+s_{2}, \chi_{v}\right)$ and hence $F_{v}\left(n^{-}, f_{v} ; s_{1}, s_{2}\right)=$ 1. 
Next suppose $v=q$ is in $S^{\prime}$, with $q^{m}$ being the conductor of $\chi_{v}$. By construction,

$$
I_{v}\left(n^{+}, f_{v} ; s_{1}, s_{2}\right)=g\left(\chi_{v}\right)^{-1} \sum_{z \in X} I_{z, v}\left(n^{+}\right)
$$

where

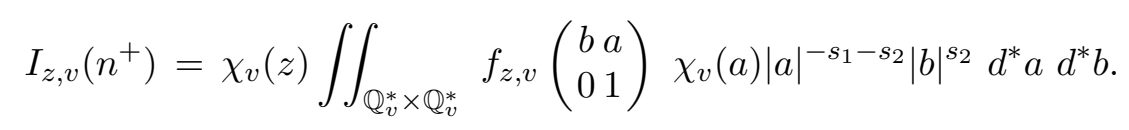

Only those $z$ with invertible images in $q^{-m} \mathbb{Z}_{q} / \mathbb{Z}_{q}$ give a non-zero contribution. So we will restrict our attention to these.

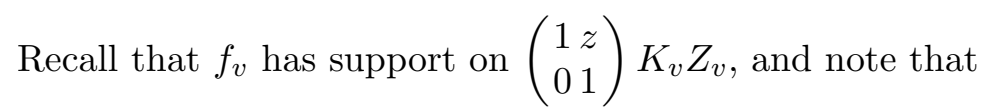

$$
\lambda\left(\begin{array}{cc}
1 & -z \\
0 & 1
\end{array}\right)\left(\begin{array}{ll}
b & a \\
0 & 1
\end{array}\right)=\left(\begin{array}{cc}
\lambda b \lambda(a-z) \\
0 & \lambda
\end{array}\right) .
$$

Then it follows that for $f_{z, v}\left(\begin{array}{ll}b & a \\ 0 & 1\end{array}\right)$ to not vanish, we need $b \in \mathbb{Z}_{q}^{*}$ and $a-z \in \mathbb{Z}_{q}$. Thus $a$ lies in $q^{-m} \mathbb{Z}_{q}$ and has the same image as $z$ in $q^{-m} \mathbb{Z}_{q} / \mathbb{Z}_{q}$. Consequently, since $\chi_{v}$ has conductor $q^{m}$ and thus the pullback of a character of $\left(q^{-m} \mathbb{Z}_{q} / \mathbb{Z}_{q}\right)^{*} \simeq$ $\left(\mathbb{Z} / q^{m}\right)^{*}$, we must have $\chi_{v}(z) \chi_{v}\left(a^{-1}\right)=1$. We obtain

$$
I_{z, v}\left(n^{+}\right)=\operatorname{vol}\left(1+q^{m} \mathbb{Z}_{q}\right)
$$

when $z$ has invertible image in $\mathbb{Z} / q^{m}$. The assertion follows once we note:

(i) $\varphi\left(q^{m}\right) \operatorname{vol}\left(1+q^{m} \mathbb{Z}_{q}\right)$ equals $\operatorname{vol}\left(\mathbb{Z}_{q}^{*}\right)=1, \quad$ and

(ii) $L\left(s, \chi_{v}\right)=1$ as $\chi_{v}$ is ramified.

Now consider the situation when $n^{+}$is replaced by $n^{-}$. We have

$$
I_{v}\left(n^{-}, f_{v} ; s_{1}, s_{2}\right)=g\left(\chi_{v}\right)^{-1} \sum_{z \in X} I_{z, v}\left(n^{-}\right)
$$

where

$$
I_{z, v}\left(n^{-}\right)=\chi_{v}(z) \iint_{\mathbb{Q}_{v}^{*} \times \mathbb{Q}_{v}^{*}} f_{z, v}\left(\begin{array}{cc}
a b & 0 \\
b & 1
\end{array}\right) \chi_{v}(a)|a|^{-s_{1}}|b|^{s_{2}} d^{*} a d^{*} b .
$$

Again, only those $z$ with invertible images in $q^{-m} \mathbb{Z}_{q} / \mathbb{Z}_{q}$ give a non-zero contribution. Write $z=q^{-m} u$, with $u \in \mathbb{Z}_{q}^{*}$. Note that

$$
\lambda\left(\begin{array}{cc}
1 & -z \\
0 & 1
\end{array}\right)\left(\begin{array}{cc}
a b & 0 \\
b & 1
\end{array}\right)=\left(\begin{array}{cc}
\lambda(a-z) b \lambda(-z) \\
\lambda b & \lambda
\end{array}\right) .
$$

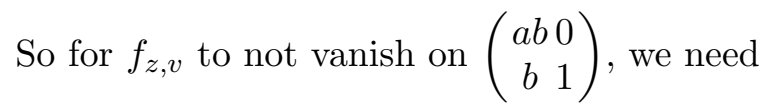

$$
\lambda^{2} a b \in \mathbb{Z}_{q}^{*}, \lambda \in \mathbb{Z}_{q}, \lambda z \in \mathbb{Z}_{q}, \lambda b(a-z) \in \mathbb{Z}_{q} .
$$


For these to hold, we must have

$$
a=v q^{-m}, b \in q^{-m} \mathbb{Z}_{q}^{*},
$$

with $v, u$ having the same image in $q^{-m} \mathbb{Z}_{q} / \mathbb{Z}_{q}$. This implies again that $\chi_{v}(z) \chi_{v}\left(a^{-1}\right)=$ 1. The assertion follows as in the $n^{+}$case. The two integrals coincide at $s_{1}=s_{2}=0$.

(b) At $v=N, f_{N}$ is the characteristic function of $Z_{N} K_{0}(N)$ divided by $V_{N}$. Thus

$$
(a, b) \rightarrow f_{N}\left(\begin{array}{ll}
b & a \\
0 & 1
\end{array}\right)
$$

is the characteristic function of $\mathbf{Z}_{N} \times \mathbf{Z}_{N}^{*}$ divided by $V_{N}$ and $I_{N}\left(n^{+}, f_{N} ; s_{1}, s_{2}\right)=$ $V_{N}^{-1} L_{v}\left(-s_{1}-s_{2}, \chi_{N}\right)$ as in (a). Similarly,

$$
(a, b) \rightarrow f_{N}\left(\begin{array}{ll}
a & 0 \\
b & 1
\end{array}\right)
$$

is the characteristic function of $\mathbb{Z}_{N}^{*} \times N \mathbb{Z}_{N}$ divided by $V_{N}$. Since $\chi$ is unramified at $N, \chi\left(a^{-1} b\right)=\chi(b)$ and a change of variables from $b$ to $N b$ gives $I_{N}\left(n^{-}, f_{N} ; s_{1}, s_{2}\right)=\chi(N)|N|^{s_{1}+s_{2}} V_{N}^{-1} L_{v}\left(s_{1}+s_{2}, \chi_{N}\right)$. Part (b) follows.

(c) Let $v=\infty$. Recall that $\chi_{\infty}(-1)=-1$. By definition,

$$
f_{\infty}\left(\left(\begin{array}{ll}
b & a \\
0 & 1
\end{array}\right)\right)= \begin{cases}2^{k} d_{k} \frac{b^{k / 2}}{(b+1+i a)^{k}} & \text { if } b>0 \\
0 & \text { if } b<0\end{cases}
$$

Noting that $d^{*} x=d x /|x|$ on $\mathbb{R}^{*}$, we obtain

$$
\begin{aligned}
2^{-k} d_{k}^{-1} I_{\infty}\left(n^{+}, f_{\infty} ; s_{1}, s_{2}\right) & =\int_{-\infty}^{\infty} \int_{0}^{\infty} \frac{b^{k / 2+s_{2}-1}|a|^{-s_{1}-s_{2}-1} \operatorname{sgn}(a)}{(b+1+i a)^{k}} d b d a \\
& =\int_{-\infty}^{\infty} \int_{0}^{\infty} \frac{b^{k / 2+s_{2}-1}|a|^{-s_{1}-s_{2}-1} \operatorname{sgn}(a)((b+1)-i a)^{k}}{\left(a^{2}+(b+1)^{2}\right)^{k}} d b d a
\end{aligned}
$$

Appealing to the binomial expansion

$$
((b+1)-i a)^{k}=\sum_{j=0}^{k}\left(\begin{array}{c}
k \\
j
\end{array}\right)(-i)^{k-j} a^{k-j}(b+1)^{j},
$$

we may write

$$
2^{-k} d_{k}^{-1} I_{\infty}\left(n^{+}, f_{\infty} ; s_{1}, s_{2}\right)=\sum_{j=0}^{k}\left(\begin{array}{c}
k \\
j
\end{array}\right)(-i)^{k-j} I_{j}
$$

where

$$
I_{j}=\int_{-\infty}^{\infty} \int_{0}^{\infty} \frac{b^{k / 2+s_{2}-1}|a|^{k-j-s_{1}-s_{2}-1}(a /|a|)^{1+k-j}(b+1)^{j}}{\left(a^{2}+(b+1)^{2}\right)^{k}} d a d b .
$$


If $j$, and hence $k-j$, is even, then the integrand is odd in the $a$-variable and $I_{j}$ vanishes. Thus we assume from hereon that $j$ is odd. We have

$$
\begin{aligned}
I_{j} & =2 \int_{0}^{\infty} \int_{0}^{\infty} \frac{b^{k / 2+s_{2}-1} a^{k-j-s_{1}-s_{2}-1}(b+1)^{j}}{\left(a^{2}+(b+1)^{2}\right)^{k}} d a d b \\
& =2 \int_{0}^{\infty} \int_{0}^{\infty} \frac{b^{k / 2+s_{2}-1} a^{k-j-s_{1}-s_{2}-1}(b+1)^{j-2 k}}{\left((a /(b+1))^{2}+1\right)^{k}} d a d b \\
& =2 \int_{0}^{\infty} \int_{0}^{\infty} \frac{b^{k / 2+s_{2}-1} a^{k-j-s_{1}-s_{2}-1}(b+1)^{-k-s_{1}-s_{2}}}{\left(a^{2}+1\right)^{k}} d a d b .
\end{aligned}
$$

So the integral factors as

$$
I_{j}=I_{j, 1} I_{j, 2}
$$

where

$$
I_{j, 1}=2 \int_{0}^{\infty} \frac{a^{k-j-s_{1}-s_{2}-1}}{\left(a^{2}+1\right)^{k}} d a
$$

and

$$
I_{j, 2}=\int_{0}^{\infty} b^{k / 2+s_{2}-1}(b+1)^{-k-s_{1}-s_{2}} d b .
$$

Note that $I_{j, 2}$ is in fact independent of $j$.

By the substitution $u=a^{2}$, we obtain

$$
I_{j, 1}=\int_{0}^{\infty} \frac{u^{\left(k-j-s_{1}-s_{2}\right) / 2-1}}{(u+1)^{k}} d u .
$$

Recall that the Beta function $B(z, w)=\frac{\Gamma(z) \Gamma(w)}{\Gamma(z+w)}$ has the following integral representations (cf. [A-S], p.258):

$$
B(z, w)=\int_{0}^{\infty} t^{z-1}(1-t)^{w-1} d t=\int_{0}^{\infty} \frac{t^{z-1}}{(1+t)^{z+w}} d t
$$

It follows immediately that

$$
I_{j, 1}=B\left(\left(k-j-s_{1}-s_{2}\right) / 2,\left(k+j+s_{1}+s_{2}\right) / 2\right)
$$

Now let $v=b /(b+1)$ in the expression for $I_{j, 2}$, so that $b=v /(1-v)$ and $d b=d v /(1-v)^{2}$. We obtain:

$$
I_{j, 2}=\int_{0}^{1} v^{k / 2-1+s_{2}}(1-v)^{k / 2+s_{1}-1} d v .
$$

Applying the first identity of (3) we obtain

$$
I_{j, 2}=B\left(k / 2+s_{2}, k / 2+s_{1}\right) .
$$


Putting (2), (4) and (5) together, and writing everything in terms of the $\Gamma$ function, we finally obtain

$$
I_{j}=\frac{\Gamma\left(\left(k-j-s_{1}-s_{2}\right) / 2\right) \Gamma\left(\left(k+j+s_{1}+s_{2}\right) / 2\right) \Gamma\left(k / 2+s_{2}\right) \Gamma\left(k / 2+s_{1}\right)}{\Gamma(k) \Gamma\left(k+s_{1}+s_{2}\right)} .
$$

We see that $2^{-k} d_{k}^{-1} I_{\infty}\left(n^{+} ; s_{1}, s_{2}\right)$ equals

$$
\left(\sum_{\substack{j=0 \\
j \equiv 1(2)}}^{k}\left(\begin{array}{c}
k \\
j
\end{array}\right)(-i)^{k-j} \Gamma\left(\frac{k-j-s_{1}-s_{2}}{2}\right) \Gamma\left(\frac{k+j+s_{1}+s_{2}}{2}\right)\right) \frac{\Gamma\left(\frac{k}{2}+s_{1}\right) \Gamma\left(\frac{k}{2}+s_{2}\right)}{\Gamma(k) \Gamma\left(k+s_{1}+s_{2}\right)} .
$$

Now observe that for any positive integer $r$,

$$
\Gamma\left(\frac{1+2 r}{2}\right)=\Gamma\left(\frac{1}{2}\right)(r-1) !=\sqrt{\pi}(r-1) !
$$

Let $k=2 m, j=2 n+1$. Then $(-i)^{k-j}=i(-1)^{m-n}$ and we see that $I_{\infty}\left(n^{+}, f_{\infty} ; 0,0\right)$ is equal to:

$$
i \pi 2^{k} d_{k} \frac{(2 m)((m-1) !)^{2}}{(2 m-1) !}\left(1+\sum_{n=0}^{m-2}\left(\begin{array}{c}
2 m \\
2 n+1
\end{array}\right)(-1)^{m-n}(m+n-1) !(m-n-2) !\right)
$$

Thus $I_{\infty}\left(n^{+}, f_{\infty} ; 0,0\right)=i \pi c_{k}$. Furthermore, since $\chi_{\infty}=$ sgn, the local factor $L\left(s, \chi_{\infty}\right)=\pi^{-(s+1) / 2} \Gamma\left(\frac{s+1}{2}\right)$ is regular at $s=0$ with value 1 . Thus $F_{\infty}\left(n^{+}, f_{\infty}\right)=$ $I_{\infty}\left(n^{+}, f_{\infty} ; 0,0\right)$ and the assertion for $F_{\infty}\left(n^{+}, f_{\infty}\right)$ follows.

The $I_{\infty}\left(n^{-}, f_{\infty} ; s_{1}, s_{2}\right)$ differs from to the expression for $I_{\infty}\left(n^{+}, f_{\infty} ; s_{1}, s_{2}\right)$ only by a change of variables $a \rightarrow-a$, which induces a sign change in the integral. In other words, $I_{\infty}\left(n^{-}, f_{\infty} ; s_{1}, s_{2}\right)=-I_{\infty}\left(n^{+}, f_{\infty} ; s_{1}, s_{2}\right)$.

The distributions appearing in our global relative trace formula are

$$
I\left(n^{ \pm}, f\right)=I\left(n^{ \pm}, f ; 0,0\right)
$$

To evaluate $I\left(n^{+}, f\right)$, we observe that $I\left(n^{+}, f ; s_{1}, s_{2}\right)$ is analytic in the region defined by the conditions $(*)$. It is defined by an absolutely convergent integral both $\left(^{*}\right)$ and the condition $\sigma_{1}+\sigma_{2}<-1$ are satisfied and in this region, we have a factorization

$$
I\left(n^{+}, f ; s_{1}, s_{2}\right)=\prod_{v} I_{v}\left(n^{+}, f_{v} ; s_{1}, s_{2}\right)=L\left(-s_{1}-s_{2}\right) \prod_{v} F_{v}\left(n^{+}, f_{v} ; s_{1}, s_{2}\right)
$$

By Proposition 2.3,

$$
I\left(n^{+}, f ; s_{1}, s_{2}\right)=g(\chi)^{-1} V_{N}^{-1} L\left(-s_{1}-s_{2}\right)\left(-i \pi c_{k}\right) F_{p}\left(n^{+}, f_{p} ; s_{1}, s_{2}\right)
$$

We set $s_{1}=s_{2}=0$ and use the functional equation

$$
L(0, \chi)=\pi^{-1}|D|^{1 / 2} L(1, \chi)=-i \pi^{-1} g(\chi) L(1, \chi)
$$


to write:

$$
I\left(n^{+}, f\right)=c_{k} L(1, \chi) F_{p}\left(n^{+}, f_{p}\right)
$$

Similarly, we obtain

$$
I\left(n^{-}, f\right)=c_{k} L(1, \chi) F_{p}\left(n^{-}, f_{p}\right)
$$

Note that in passing from $n^{+}$to $n^{-}$, the local integral at $v=N$ changes sign since $\chi(N)=-1$. This compensates for a similar change of sign at $v=\infty$.

Corollary 1. We have

$$
I\left(n^{+}, f\right)+I\left(n^{-}, f\right)=c_{k} V_{N}^{-1} L(1, \chi)\left(F_{p}\left(n^{+}, f_{p}\right)+F_{p}\left(n^{-}, f_{p}\right)\right)
$$

2.7. The regular terms. Recall that the regular double cosets are represented by the matrices $\{\xi(x)\}$, with $x \in \mathbb{P}^{1}(\mathbb{Q})-\{0,1, \infty\}$. By abuse of notation we will write $I(x)$ for $I(\xi(x))$. At any place $v$, we have by definition,

$$
I_{v}(x)=\int_{\mathbb{Q}_{v}^{*} \times \mathbb{Q}_{v}^{*}} f_{v}\left(\begin{array}{cc}
a b a x \\
b & 1
\end{array}\right) \chi_{v}(a)^{-1}|a|^{-s_{1}}|b|^{s_{2}} d^{*} a d^{*} b
$$

Set

$$
\rho=k / 2-s_{1}, \quad \text { and } \quad \sigma=k / 2+s_{2} .
$$

For $\epsilon, \delta, \nu \in\{ \pm 1\}$, define

$I_{\infty}(\epsilon, \delta, \nu)=(-\epsilon)^{\rho-\sigma-1} \delta^{\rho+k-3 \sigma-1} \nu^{k-\sigma} i^{\rho-2 \sigma} B(\sigma, k-\sigma) B(\rho, k-\rho) F(k-\sigma, \rho ; k ; 1-\epsilon \nu)$

where $F={ }_{2} F_{1}$ is the hypergeometric function.

Proposition 2.4. Let $x \in \mathbb{P}^{1}(\mathbb{Q})-\{0,1, \infty\}$.

(a): Let $v=q$ be a prime not in $S$. When $v(1-x)>0, I_{v}(x)$ vanishes. Suppose $v(1-x)=0$, resp. $v(1-x)<0$. Then $v(x) \geq 0$, resp. $v(x)=$ $v(1-x)$, and we have:

$$
\left.I_{v}(x)=\sum_{n=0}^{v(x)} \sum_{m=-v(x)}^{-n} '\left(\bar{\chi}(q) q^{s_{1}}\right)^{m} q^{-n s_{2}}\right),
$$

resp.

$$
I_{v}(x)=\sum_{n=v(x)}^{0} \sum_{m=0}^{-n}{ }_{m}^{\prime}\left(\bar{\chi}(q) q^{s_{1}}\right)^{m} q^{-n s_{2}},
$$

where the prime on the inside sum implies (in either case) that the summation is taken over $m$ of the same parity as $n+v(1-x)$. In particular, since $\operatorname{vol}\left(\mathbb{Z}_{v}^{*}\right)=1$,

$$
v(x)=v(1-x)=0 \Longrightarrow I_{v}(x)=1 .
$$


(b): Let $v=q$ be a prime in $S^{\prime}$, with $q^{c}$ the conductor of $\chi_{q}$. Then if $\left.v(1-x)>c, I_{v}(x, f)\right)$ vanishes. When $v(1-x) \leq c, I_{v}(x, f)$ is bounded by $q^{c / 2}(v(x)+c)^{2}$.

(c): Let $v=N$. Then $I_{N}(x)$ vanishes unless $v_{N}(1-x)=0$ and $v_{N}(x) \geq 1$, and in this case,

$$
I_{N}(x)=\sum_{n=1}^{v_{N}(x)} \sum_{m=-v_{N}(x)}^{-n}{ }^{-n}\left(\bar{\chi}(N) N^{s_{1}}\right)^{m} N^{-n s_{2}} .
$$

(d): Let $v=p$, and $f_{p}$ the characteristic function of $Z K\left(\begin{array}{cc}p^{r} & 0 \\ 0 & p^{r^{\prime}}\end{array}\right) K$ for some integers $r, r^{\prime}$ such that $r \geq r^{\prime}$. Then $I_{p}(x, f)$ is zero unless $v(1-x)<$ $r+r^{\prime}$, in which case it is bounded by $C\left(f_{p}\right) v(x)^{2}$, for a positive constant $C\left(f_{p}\right)$.

(e): Let $v=\infty$. Assume that $x>0$ and that $\sigma_{1}, \sigma_{2} \in(-k / 2, k / 2)$. Then, as $\chi_{\infty}(-1)=-1$, if $1-x>0$ we have

$$
I_{\infty}(x)=(1-x)^{k / 2}\left(I_{\infty}(-1,1,1)-(-1)^{k} I_{\infty}(-1,-1,1)\right)
$$

and if $1-x<0$ then

$$
I_{\infty}(x)=(x-1)^{k / 2}\left(I_{\infty}(1,-1,-1)-(-1)^{k} I_{\infty}(1,1,-1)\right),
$$

Proof. (a) Since $v=q$ is a prime outside $S, f_{v}$ is by definition the characteristic function of $Z_{v} K_{v}$.

Lemma 5. $f_{v}\left(\begin{array}{cc}a b & a x \\ b & 1\end{array}\right)$ is non-zero iff the following conditions are simultaneously satisfied:

(i) $v(1-x) \leq 0$;

(ii) $v(x) \geq v(1-x)$; in particular, $v(x)=v(1-x)$ when $v(1-x)<0$;

(iii) $v(1-x)-v(x) \leq v(a) \leq \min \{-v(1-x),-v(b)-v(1-x)\}$;

(iv) $\max \{v(1-x), v(a)+v(1-x)\} \leq v(b) \leq v(x)-v(1-x)$;

(v) $v(a)+v(b) \equiv v(1-x) \quad(\bmod 2)$.

Proof of Lemma. For $f_{v}\left(\begin{array}{cc}a b a x \\ b & 1\end{array}\right)$ to be non-zero, it is necessary and sufficient that there exists a $\lambda$ in $\mathbb{Q}_{v}^{*}$ such that $\lambda a b, \lambda a x, \lambda b, \lambda$ are in $\mathbb{Z}_{v}$ and $\lambda^{2} a b(1-x)$ is in $\mathbb{Z}_{v}^{*}$; in other words, one must have

$$
2 v(\lambda)+v(a)+v(b)+v(1-x)=0 ;
$$

$$
v(\lambda)+v(a)+v(b) \geq 0
$$




$$
\begin{gathered}
v(\lambda)+v(a)+v(x) \geq 0 \\
v(\lambda)+v(b) \geq 0
\end{gathered}
$$

and

$$
v(\lambda) \geq 0 .
$$

Eliminating $v(\lambda)$ from these, we arrive at the following system of inequalities, together with the parity condition

$$
v(a)+v(b)+v(1-x) \equiv 0(\bmod 2):
$$

$$
v(a)+v(x)-v(1-x) \geq 0
$$

$$
\begin{gathered}
v(b)-v(1-x) \geq 0 ; \\
v(x)-v(1-x) \geq 0 ; \\
v(1-x) \leq 0 ; \\
v(a)+v(1-x) \leq 0 ; \\
v(b)+v(1-x)-v(x) \leq 0 ; \\
v(a)+v(b)+v(1-x) \leq 0,
\end{gathered}
$$

and

$$
v(b) \geq v(a)+v(1-x) .
$$

To explain, 6) comes from 2) +3 ) -1 ), while 7) comes from 2) +4 ) -1 ), 8) from $3)+4)-1$ ), 9) from 1) -2 ) -5 ), 10) from 1) - 4) - 5), 11) from 1) - 3) - 5), 12) from 1) $-2 \times 5$ ), and 13) from $2 \times 4$ ) -1 ). The assertions of the Lemma now follows easily.

The first consequence is that $I_{v}(x)$ vanishes if $v(1-x)$ is positive. Now let $v(1-x)$ be zero (resp. negative). Then $v(x) \geq 0$ (resp. $v(x)=v(1-x))$ and the inequalities (iii), (iv) of the Lemma simplify to yield the conditions

$$
0 \leq v(b) \leq v(x), \quad \text { and } \quad-v(x) \leq v(a) \leq-v(b)
$$

(resp.

$$
v(x) \leq v(b) \leq 0 \quad \text { and } \quad 1 \leq v(a) \leq-v(x) .)
$$

The assertion of the Proposition now follows in the case $v=q \notin S$.

(b) Here $v=q$ is a prime where $\chi$ ramifies. If the conductor of $\chi_{v}$ is $q^{c}$, with $c \geq 1, f_{v}$ is, by definition, $g\left(\chi_{v}\right)^{-1}$ times a $\chi$-weighted sum of the characteristic 
functions $f_{z, v}$ of $\left(\begin{array}{c}1 \\ z \\ 0\end{array}\right) K_{v} Z_{v}$, where $z$ has valuation $-r$ with $0 \leq r \leq m$. We need the following:

Lemma 6. $f_{z, v}\left(\begin{array}{cc}a b & a x \\ b & 1\end{array}\right)$ is non-zero only if the following conditions are simultaneously satisfied:

(i) $v(1-x) \leq r$;

(ii) $v(x)=v(1-x)$ when $v(1-x)<0$, and $v(x)=0$ when $v(1-x)>0$;

(iii) $v(a) \leq \min \{-v(1-x),-v(b)-v(1-x)\}, v(a+z)+v(a x+z)-v(a) \geq$ $v(1-x)$;

(iv) $\max \{v(1-x)+v(a), v(1-x)-v(a+z)+v(a)\} \leq v(b) \leq v(x)-v(1-x)$;

(v) $v(a)+v(b) \equiv v(1-x) \quad(\bmod 2)$.

Proof of Lemma. The statement for $r=0$ is just Lemma 5. So we may assume that $0<r \leq c$. Then

$$
\left(\begin{array}{cc}
1 & z \\
0 & 1
\end{array}\right)\left(\begin{array}{cc}
a b a x \\
b & 1
\end{array}\right)=\left(\begin{array}{cc}
a b+b z & a x+z \\
b & 1
\end{array}\right)
$$

So for $f_{r, v}\left(\begin{array}{cc}a b a x \\ b & 1\end{array}\right)$ to be non-zero, it is necessary and sufficient that there exists a $\lambda$ in $\mathbb{Q}_{v}^{*}$ such that $\lambda(a+z) b, \lambda(a x+z), \lambda b, \lambda$ are in $\mathbb{Z}_{v}$ and $\lambda^{2} a b(1-x)$ is in $\mathbb{Z}_{v}^{*}$. In other words, one must have

$$
\begin{gathered}
2 v(\lambda)+v(a)+v(b)+v(1-x)=0 \\
v(\lambda)+v(a+z)+v(b) \geq 0 \\
v(\lambda)+v(a x+z)) \geq 0 \\
v(\lambda)+v(b) \geq 0
\end{gathered}
$$

and

$$
v(\lambda) \geq 0
$$

Eliminating $v(\lambda)$ from these, we arrive at the following system of inequalities, together with the parity condition

$$
v(a)+v(b)+v(1-x) \equiv 0(\bmod 2):
$$

$$
\begin{gathered}
v(a+z)+v(a x+z)-v(a)-v(1-x) \geq 0 \\
v(b)+v(a+z)-v(a)-v(1-x) \geq 0 \\
v(a x+z)-v(a)-v(1-x) \geq 0
\end{gathered}
$$




$$
\begin{gathered}
v(1-x) \leq v(a+z)-v(a) \\
v(a)+v(1-x) \leq 0 ; \\
v(b)+v(1-x)-v(x) \leq 0 ; \\
v(a)+v(b)+v(1-x) \leq 0 ;
\end{gathered}
$$

and

$$
v(b) \geq v(a)+v(1-x) .
$$

To explain, $6^{\prime}$ ) comes from $\left.\left.2^{\prime}\right)+3^{\prime}\right)-1^{\prime}$ ), while $7^{\prime}$ ) comes from $\left.\left.\left.2^{\prime}\right)+4^{\prime}\right)-1^{\prime}\right), 8^{\prime}$ ) from $\left.\left.3^{\prime}\right)+4^{\prime}\right)-1^{\prime}$ ), $9^{\prime}$ ) from $\left.\left.\left.1^{\prime}\right)-2^{\prime}\right)-5^{\prime}\right), 10^{\prime}$ ) from $\left.\left.\left.1^{\prime}\right)-4^{\prime}\right)-5^{\prime}\right), 11^{\prime}$ ) from $\left.\left.\left.1^{\prime}\right)-3^{\prime}\right)-5^{\prime}\right), 12^{\prime}$ ) from $\left.\left.1^{\prime}\right)-2 \times 5^{\prime}\right)$, and $\left.13^{\prime}\right)$ from $\left.\left.2 \times 4^{\prime}\right)-1^{\prime}\right)$. If $v(a) \neq v(z)$, then $v(a+z)=\min \{v(a), v(z)\}$, and so $v(a+z)-v(a) \leq 0$, and by $\left.9^{\prime}\right), v(1-x) \leq 0$. On the other hand, if $v(a)=v(z)=-r$, so $\left.10^{\prime}\right)$ implies that $v(1-x) \leq r$. This gives part (i) of the Lemma. The remaining assertions follow easily.

So the first consequence is that $I_{v}(x)$ vanishes if $v(1-x)$ is greater than $r$. We get from the Lemma:

$$
v(1-x)-r \leq v(b) \leq v(x)-v(1-x) .
$$

This is clear when $v(a)=-r$, and if not, $v(a+z)-v(a) \leq 0$, which even gives $v(1-x) \leq v(b)$. Next observe that when $v(1-x)$ is 0 , resp. $<0$, resp. $>0$, we have $v(x) \geq 0$, resp. $v(x)=v(1-x)$, resp. $v(x)=0$. We obtain

$$
-r \leq v(b) \leq v(x) \text { and } \quad-v(x) \leq v(a) \leq 0,
$$

resp.

$$
v(x)-r \leq v(b) \leq 0 \quad \text { and } \quad 0 \leq v(a) \leq-v(x) \quad \text { or } \quad v(a)=-r-v(x),
$$

resp.

$$
-r<v(b)<0 \quad \text { and } \quad v(a)=-r
$$

The assertion of the Proposition now follows in the case $v=q \in S^{\prime}$, once we recall that $\left|g\left(\chi_{v}\right)\right|=q^{c / 2}$.

(c) Let $v=N$. Here by construction, $f_{N}$ is the characteristic function of $Z_{N} K_{0}(N)$ divided by $V_{N}$, the volume of $Z_{N} K_{0}(N) / Z_{N}$. So for $f_{N}\left(\left(\begin{array}{cc}a b a x \\ b & 1\end{array}\right)\right)$ to be non-zero, it is necessary and sufficient that there exists a $\lambda$ in $\mathbb{Q}_{N}^{*}$ such that $\lambda a b, \lambda a x, \lambda$ are in $\mathbb{Z}_{N}, \lambda b$ is in $N \mathbb{Z}_{N}$ and $\lambda^{2} a b(1-x)$ is in $\mathbb{Z}_{N}^{*}$. The conditions 1$)$ through 5) above (in the proof of part (a)) remain in force except for 4 ), which gets replaced by

$\left.4^{\prime \prime}\right)$

$$
v_{N}(\lambda)+v_{N}(b) \geq 1
$$


The parity condition is the same as in (a). The only change in the inequalities $6)-12$ ) is that 7) (resp. 8), resp. 10)) gets replaced by

$\left.7^{\prime \prime}\right)$

$\left.8^{\prime \prime}\right)$

and

$\left.11^{\prime \prime}\right)$

$$
\begin{gathered}
v_{N}(b)-v_{N}(1-x) \geq 1 \\
v(x)-v(1-x) \geq 1
\end{gathered}
$$

We again get the vanishing of $I_{N}(x)$ when $v_{N}(1-x)>0$. Moreover, when $v_{N}(1-x)<0$, we are forced to have $v_{N}(x)=v_{N}(1-x)$, which contradicts $\left.7^{\prime}\right)$; thus $I_{N}(x)$ vanishes in this case as well. It remains only to consider when $v_{N}(1-x)=0$, in which case $v_{N}(x) \geq 1$ by $\left.8^{\prime \prime}\right)$. The asserted expression for $I_{N}(x)$ follows easily.

(d) Of course, when $r=r^{\prime}=0, f_{p}$ is just the characteristic function of $Z_{p} K_{p}$, which was treated in detail in part (a). For general $r, r^{\prime}$ with $r \geq r^{\prime}$, for $f_{p}\left(\begin{array}{cc}a b & a x \\ b & 1\end{array}\right)$ to be non-zero, it is necessary and sufficient that there exists a $\lambda$ in $\mathbb{Q}_{v}^{*}$ such that

$$
\left(\begin{array}{cc}
\lambda a b \lambda a x \\
\lambda b & \lambda
\end{array}\right) \in K_{p}\left(\begin{array}{cc}
p^{r} & 0 \\
0 & p^{r^{\prime}}
\end{array}\right) K_{p}
$$

We get analogues of conditions 1) through 12) of part (a), except for replacing the zeros on the right of those equations. In 1) and 9), replace 0 by $r+r^{\prime}$, while in 2) through 4), replace 0 by $r$. The assertions of part d) now follow easily.

(e) Let $v=\infty$. By hypothesis, $\chi_{\infty}$ is $s g n$, the sign character. By the definition of $f_{\infty}$, we have

$$
f_{\infty}\left(\begin{array}{cc}
a b a x \\
b & 1
\end{array}\right)=\frac{(a b(1-x))^{k / 2}}{(a x-b+i(a b-1))^{k}} \quad(\text { resp.0) }
$$

if $a b(1-x)$ is $>0$ (resp. $<0)$.

Suppose $1-x>0$. Then the integral is over the first and the third quadrants. Changing variables in the third quadrant and rearranging, we get

$$
I_{\infty}(x)=(1-x)^{k / 2}\left(I_{\infty}^{\prime}(-1,1,1)-(-1)^{k} I_{\infty}^{\prime}(-1,-1,1)\right),
$$

where for $\epsilon, \delta, \nu \in\{ \pm 1\}$,

$$
I_{\infty}^{\prime}(\epsilon, \delta, \nu)=\int_{0}^{\infty} \int_{0}^{\infty} \frac{a^{k / 2-s_{1}-1} b^{k / 2+s_{2}-1} d a d b}{(a x+\epsilon b+\delta i(a b+\nu))^{k}} .
$$

Similarly, when $1-x$ is negative, we have

$$
I_{\infty}(x)=(x-1)^{k / 2}\left(I_{\infty}^{\prime}(1,-1,-1)-(-1)^{k} I_{\infty}^{\prime}(1,1,-1)\right) .
$$


Then part (c) of Proposition 2.4 will follow once we establish the following, which was shown to us by Nathaniel Grossman. Recall that

$$
\rho=k / 2-s_{1}, \quad \text { and } \quad \sigma=k / 2+s_{2} .
$$

Lemma 7. Suppose $k>\Re \rho>0$ and $k>\Re \sigma>0$. Then we have

$$
I_{\infty}^{\prime}(\epsilon, \delta, \nu)=I_{\infty}(\epsilon, \delta, \nu)
$$

with $I_{\infty}(\epsilon, \delta, \nu)$ as defined in Proposition 2.4 (e). This holds in the complex $x$ plane with the negative $x$-axis cut out.

Proof. Fix $\epsilon, \delta, \nu$ and simply write $I^{\prime}$ for $I_{\infty}^{\prime}(\epsilon, \delta, \nu)$. We have

$$
\begin{aligned}
I^{\prime} & =\int_{0}^{\infty} a^{\rho-1} d a \int_{0}^{\infty} \frac{b^{\sigma-1} d b}{((a x+\delta \nu i)+b(\delta i a+\epsilon))^{k}} \\
& =\int_{0}^{\infty} \frac{a^{\rho-1} d a}{(\delta i a+\epsilon)^{k}} \int_{0}^{\infty} \frac{b^{\sigma-1} d b}{\left(\left(\frac{a x+\delta \nu i}{\delta i a+\epsilon}\right)+b\right)^{k}} \\
& =\int_{0}^{\infty} \frac{a^{\rho-1}\left(\frac{a x+\delta \nu i}{\delta i a+\epsilon}\right)^{\sigma} d a}{(\delta i a+\epsilon)^{k}\left(\frac{a x+\delta \nu i}{\delta i a+\epsilon}\right)^{k}} \int_{L} \frac{b_{1}^{\sigma-1} d b_{1}}{\left(1+b_{1}\right)^{k}},
\end{aligned}
$$

where $L$ denotes the half-line defined by the positive real multiples of $\frac{\delta i a+\epsilon}{a x+\delta \nu i}$.

Since $k>\Re \sigma>0$ by hypothesis, the ray of integration may be rotated back to $\mathbb{R}_{+}$, and the inner $b_{1}$-integral becomes

$$
\int_{0}^{\infty} \frac{b_{1}^{\sigma-1} d b_{1}}{\left(1+b_{1}\right)^{k}}=B(\sigma, k-\sigma) .
$$

Thus

$$
I^{\prime}=\frac{B(\sigma, k-\sigma)}{x^{k-\sigma}(\delta i)^{\sigma}} J
$$

where

$$
J=\int_{0}^{\infty} \frac{a^{\rho-1} d a}{(a+\delta \nu i / x)^{k-\sigma}(a-i \delta \epsilon)^{\sigma}}
$$


Since by assumption $k>\Re \rho>0$, the path of integration defining $J$ can be rotated to the positive imaginary axis, giving

$$
\begin{aligned}
J & =\int_{0}^{i \infty} \frac{a^{\rho-1} d a}{(a+\delta \nu i / x)^{k-\sigma}(a-i \delta \epsilon)^{\sigma}} \\
& =\int_{0}^{\infty} \frac{(i c)^{\rho-1} d(i c)}{(i c+\delta \nu i / x)^{k-\sigma}(i c-i \delta \epsilon)^{\sigma}} \\
& =i^{\rho-\sigma} \int_{0}^{\infty} \frac{c^{\rho-1} d c}{(c+\delta \nu / x)^{k-\sigma}(c-\delta \epsilon)^{\sigma}}
\end{aligned}
$$

Case (i): $\delta \epsilon=-1$.

Put $u=\frac{c}{1+c}$ so that $c=\frac{u}{1-u}$ and $d c=\frac{d u}{(1-u)^{2}}$. We get

$$
J=i^{\rho-\sigma}(\delta \nu)^{k-\sigma} x^{k-\sigma} \int_{0}^{1} \frac{u^{\rho-1}(1-u)^{k-\rho-1} d u}{(1-u(1-\delta \nu x))^{k-\sigma}} .
$$

Now we appeal to the following well known integral representation for the hypergeometric function ([A-S], formula (15.3.1), page 558):

$$
F(a, b ; c ; z)=\frac{\Gamma(c)}{\Gamma(b) \Gamma(c-b)} \int_{0}^{1} t^{b-1}(1-t)^{c-b-1}(1-t z)^{-a} d t,
$$

for $\Re c>\Re b>0$.

The assertion follows by putting these together.

Case (ii): $\quad \delta \epsilon=1$.

In this case,

$$
J=i^{\rho-\sigma} \int_{0}^{\infty} \frac{c^{\rho-1} d c}{(c+\delta \nu / x)^{k-\sigma}(c-1)^{\sigma}} .
$$

We put $c=\frac{u}{u-1}$, so that $u=\frac{c}{c-1}$ and $d c=\frac{-d u}{(1-u)^{2}}$. We get

$$
J=i^{\rho-\sigma}(\delta \nu)^{k-\sigma} x^{k-\sigma}(-1)^{\rho-\sigma-1} \int_{0}^{1} \frac{u^{\rho-1}(1-u)^{k-\rho-1} d u}{(1-u(1+\delta \nu x))^{k-\sigma}} .
$$

The assertion follows by appealing once again to the integral representation of the hypergeometric function and combining like terms.

\section{A bound for the sum of regular terms}

Put

$$
I_{\mathrm{reg}}(f):=\sum_{x \in \mathbb{Q}^{*}-\{1\}} I(x, f)
$$


Proposition 3.1. Let $\varepsilon>0$. Then

$$
I_{\mathrm{reg}}(f) \leq \frac{C}{N^{k / 2-\varepsilon}}
$$

for a positive constant $C$.

Proof. . Put $t=\frac{1}{1-x}$. Since $x \rightarrow t$ is an automorphism of $\mathbb{Q}^{*}-\{1\}$,

$$
I_{\mathrm{reg}}(f):=\sum_{t \in \mathbb{Q}^{*}-\{1\}} I(x, f),
$$

with $x=\frac{t-1}{t}$. By Proposition 2.4, there is a positive integer $M=D p^{r}$ such that for any finite place $v$,

$$
v(t)<-v(M) \Longrightarrow I_{v}(x, f)=0 .
$$

In fact, Proposition 2.4 implies that for $v=N, I_{v}(x, f) \neq 0 \Longrightarrow N \mid(t-1)$. Also, $I_{\infty}(x, f)=0$ unless $x>0$. Putting these together, we see that $I(x, f)$ is zero unless $N \mid(t-1)$ and $M t$ is an integer $\neq 0,1$. Thus

$$
I_{\text {reg }}(f):=\sum_{n \neq 0,1, N \mid(n-M)} I\left(\frac{n-M}{n}, f\right) .
$$

Lemma 8. For any $n \neq 0$,

$$
I\left(\frac{n-M}{n}, f\right)<<\frac{1}{n^{k / 2-\varepsilon}},
$$

with the implied constant independent of $n$,

Proof. By Prop. 2.4, for any (finite) prime $q, I_{q}\left(\frac{n-M}{n}, f\right)$ is 1 for $q \nmid n(n-M)$, and if $q \mid n(n-M)$,

$$
I_{q}\left(\frac{n-M}{n}, f\right) \leq M v_{q}((n-M) / n)^{2} .
$$

We claim that the function

$$
g(n):=\prod_{q} v_{q}(n)
$$

satisfies, for every $\varepsilon>0$, the bound

$$
g(n) \leq C n^{\varepsilon}
$$

for a constant $C>0$ independent of $n$.

To see this, first note that $g(n)$ is multiplicative in $n$ (though not strictly). Fix any $\varepsilon>0$. Then $\exists A>0$ such that for any prime $q$ and positive integer $a>0$,

$$
a \leq q^{a \varepsilon} \text { if either } a>A \text { or } q>A .
$$


Given $n>0$ with unique factorization $n=\prod_{j \in J} q_{j}^{a_{j}}$, where the $q_{j}$ are primes and $a_{j}>0$, we may write $n=m k$, where $m$ has no prime divisors $q_{j}<A$ with exponents $a_{j}<A$. It follows immediately that $g(m) \leq n m^{\varepsilon}$, and $k$ is a product over primes $\leq A$ with exponents at most $A$. We obtain

$$
g(n)=g(m) g(k) \leq C n^{e}
$$

where $C$ is the maximum of $g(k)$ for the finitely many choices of $k$.

Hence the claim.

It follows that for any $\varepsilon>0$,

$$
\prod_{q \mid n(n-M)} v_{q}((n-M) / n)^{2}<<n^{\varepsilon} .
$$

Moreover, thanks to Prop. 2.4, we have

$$
I_{\infty}\left(\frac{n-M}{n}, f\right)<<\frac{1}{n^{k / 2}} .
$$

In both estimates, the implied constants depends on $M$, but not on $N$. This proves the Lemma.

Consequently,

$$
I_{\text {reg }}(f)<<\sum_{n \neq 0,1, N \mid n-M} \frac{1}{n^{k / 2-\varepsilon}} .
$$

But for any real $u>1$,

$$
\sum_{n \neq 0,1, N \mid n-M} \frac{1}{n^{u}}=\sum_{m \geq 1} \frac{1}{(m N+M)^{u}}<<\frac{1}{N^{u}} .
$$

The Proposition now follows.

\section{The spectral side}

Let $\rho$ denote the right regular representation of $\widetilde{G}(\mathbb{A})$ on the space of cusp forms on $\widetilde{G}(\mathbb{Q}) \backslash \tilde{G}(\mathbb{A})$ and let $S_{N, \mathbb{A}}(k)$ be the space of adelic, holomorphic cusp forms of weight $k$ which are right-invariant under the open compact subgroup $\widetilde{K}=\widetilde{K}_{0}(N) \times \prod_{v \neq N} \widetilde{K}_{v}$. Recall that there is identification

$$
\widetilde{G}(\mathbb{Q}) \backslash \widetilde{G}(\mathbb{A}) / \widetilde{K}=\Gamma_{0}(N) \backslash \mathcal{H}
$$

induced by the map $g_{\infty} \times 1 \rightarrow g_{\infty}(i)$ for $g_{\infty}=\left(\begin{array}{ll}a & b \\ c & d\end{array}\right)$ in $\widetilde{G}_{\infty}$ of positive determinant. This yields an identification $\psi \leftrightarrow \varphi$ of the spaces $S_{N, \mathbb{A}}(k)$ and $S_{N}(k)$ such 
that

$$
\psi\left(g_{\infty} \times 1\right)=\operatorname{det}\left(g_{\infty}\right)^{k / 2} j\left(g_{\infty}, i\right)^{-k} \varphi\left(g_{\infty}(i)\right)
$$

where $j\left(g_{\infty}, z\right)=c z+d$ as usual. On $S_{N, \mathbb{A}}(k)$ we have the adelic scalar product:

$$
\left\langle\psi_{1}, \psi_{2}\right\rangle_{\mathbb{A}}=\int_{\widetilde{G}(\mathbb{Q}) \backslash \widetilde{G}(\mathbb{A})} \psi_{1}(g) \overline{\psi_{2}(g)} d g
$$

We take $d g=d g_{\infty} \times d g_{0}$ where $d g_{\infty}$ is the Haar measure on $\widetilde{G}_{\infty}$ whose pushforward to the upper half-plane $\mathcal{H}$ coincides with the measure used to define the Petersson norm on $S_{N}(k)$ (for example, $y^{-2} d x d y$ ) and $d g_{0}$ is the Haar measure on $\widetilde{G}\left(\mathbb{A}_{f}\right)$ giving measure one to $\prod_{v \neq \infty} \widetilde{K_{v}}$. Then

$$
\left\langle\psi_{1}, \psi_{2}\right\rangle_{\mathbb{A}}=V_{N}\left(\varphi_{1}, \varphi_{2}\right)
$$

where $\psi_{i}$ corresponds to $\varphi_{i}$.

Let $\rho_{0}$ denote the action of $\tilde{G}\left(\mathbb{A}_{f}\right)$ where $\mathbb{A}_{f}$ is the ring of finite adeles. Write $f=f_{\infty} \otimes f_{0}$, where $f_{0}$ denotes the finite part of $f$. Recall that $f_{\infty}$ is equal to $d_{k}$ times the complex conjugate of the matrix coefficient of $\mathcal{D}_{k}$ of weight $k$ and that $f_{0}$ is bi- $K$-invariant. Since $k>2, f_{\infty}$ is integrable. Therefore it acts as a projection operator onto the subspace of holomorphic cusp forms of weight $k$ and the image of $\rho_{0}(f)$ is contained in $S_{N, \mathbb{A}}(k)$. It follows that the kernel has the expression

$$
K_{f}(x, y)=\sum_{j} \frac{\left(\rho_{0}\left(f_{0}\right) \psi_{j}\right)(x) \overline{\psi_{j}(y)}}{\left\langle\psi_{j}, \psi_{j}\right\rangle_{\mathbb{A}}}
$$

where the sum is over an orthogonal basis of $S_{N, \mathbb{A}}(k)$. We may assume that the $\psi_{j}$ are Hecke eigenforms, i.e., generate an admissible representation $\pi_{j}=\otimes \pi_{j, v}$ of $\operatorname{GL}(2, \mathbb{A})$ satisfying $\pi_{j, \infty} \simeq \mathcal{D}_{k}$ and $\pi_{j}^{\widetilde{K}} \neq 0$.

From the expression for the kernel in terms of cusp forms, it follows that the distribution $I\left(f ; s_{1}, s_{2}\right)$ converges conditionally for all $s_{1}$ and $s_{2}$. The value $I(f)=I(f ; 0,0)$ is therefore defined.

Recall that to every $x \in[-2,2]$ there is a unique unramified representation $\pi_{x}$ of $\widetilde{G}_{p}$ with Satake parameters $\left\{p^{s}, p^{-s}\right\}$ defined by $x=p^{s}+p^{-s}$. For $f_{p} \in \mathcal{H}_{p}$, let write $f_{p}^{\wedge}(x)$ for the eigenvalue of $f_{p}$ acting on the $\widetilde{K}_{p}$-invariant vector in $\pi_{x}$. As is well-known, $f_{p}^{\wedge}(x)$ is a polynomial in $x$. In particular, for each $j, \pi_{j, p}$ is the unramified representation $\pi_{x}$ where $x=a_{p}\left(\varphi_{j}\right)$ is the normalized $p$-Hecke eigenvalue of $\varphi_{j}$.

Proposition 4.1. , We have

$$
I(f)=\frac{1}{V_{N}} \sum_{j} \frac{L\left(1 / 2, \varphi_{j}\right) L\left(1 / 2, \varphi_{j} \otimes \chi\right)}{\left(\varphi_{j}, \varphi_{j}\right)} f_{p}^{\wedge}\left(a_{p}\left(\varphi_{j}\right)\right),
$$


Proof. Using the expression above for the kernel in conjunction with the definition of $I(f)$, we obtain

$$
I\left(f ; s_{1}, s_{2}\right)=\sum_{j} P_{j}\left(f, \chi, s_{1}\right) Q_{j}\left(s_{2}\right)
$$

where

$$
P_{j}\left(f, \chi, s_{1}\right)=\int_{\mathbb{Q}^{*} \backslash \mathbb{A}^{*}} \rho(f) \psi_{j}\left(\begin{array}{ll}
a & 0 \\
0 & 1
\end{array}\right) \chi(a)|a|^{s_{1}} d^{*} a
$$

and

$$
Q_{j}\left(s_{2}\right)=\int_{\mathbb{Q}^{*} \backslash \mathbb{A}^{*}} \bar{\psi}_{j}\left(\begin{array}{ll}
b & 0 \\
0 & 1
\end{array}\right)|a|^{s_{2}} d^{*} b .
$$

If $W_{j}$ denotes the Whittaker function of $\psi_{j}$, there is a well-known Fourier expansion (for $g \in \mathrm{GL}(2, \mathbb{A})$ )

$$
\psi_{j}(g)=\sum_{t \in \mathbb{Q}^{*}} W_{j}\left[\left(\begin{array}{ll}
t & 0 \\
0 & 1
\end{array}\right) g\right] .
$$

Since $\chi$ and the adelic absolute value $|\cdot|$ are 1 on $\mathbb{Q}^{*}$, we can unfold the expression for $P_{j}$ as

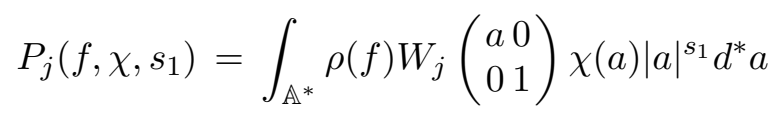

Then from the factorizability of $W_{j}, f$ and $\chi$, we obtain the product expansion

$$
P_{j}\left(f, \chi, s_{1}\right)=\prod_{v} P_{j, v}\left(f, \chi, s_{1}\right),
$$

where $v$ runs over all the places of $\mathbb{Q}$, with

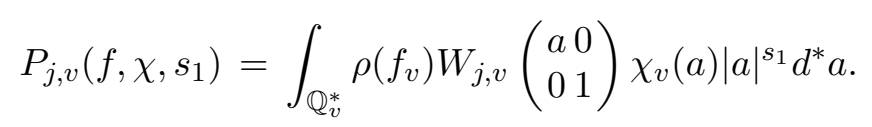

For $v \neq p, f_{v}$ is chosen such that $\rho\left(f_{v}\right) W_{j, v}$ times $\chi_{v} \circ$ det is the new vector of $\pi_{j, v} \otimes \chi_{v}$, so that by Jacquet-Langlands,

$$
P_{j, v}\left(f, \chi, s_{1}\right)=L\left(s_{1}+1 / 2, \pi_{j, v} \otimes \chi_{v}\right) .
$$

This is clear at any $v$ where $\chi$ is unramified. Let us indicate the reason at a $v=q$ where $\chi$ is ramified, say of conductor $q^{m}$. It follows from the definition of $f_{v}$, and the transformation property of the Whittaker function under the left translation by the maximal unipotent subgroup, that

$$
P_{j, v}\left(f, \chi, s_{1}\right)=\frac{1}{g\left(\chi_{v}\right)} \sum_{n \in \mathbb{Z}} W_{v}\left(\begin{array}{cc}
q^{n} & 0 \\
0 & 1
\end{array}\right) g\left(\chi_{v}, \psi_{q^{n-m}}\right) q^{-n\left(s_{1}+1 / 2\right)},
$$


where $\psi_{t}(x)=\psi(t x)$ and $\psi$ the additive character defined in section 2.2. Note that $g\left(\chi_{v}\right)$ is just $g\left(\chi_{v}, \psi_{q^{-m}}\right)$. Since $W_{j, v}$ has been chosen to be the spherical vector giving the right $L$-factor of $\pi_{v}$, we have

$$
W_{j, v}\left(\begin{array}{cc}
q^{n} & 0 \\
0 & 1
\end{array}\right)=\delta_{n \geq 0} q^{n / 2}\left(\frac{\alpha^{n+1}-\alpha^{-n-1}}{\alpha-\alpha^{-1}}\right),
$$

where $\delta_{n \geq 0}$ is 1 if $n \geq 0$ and is zero otherwise, and $\left\{\alpha, \alpha^{-1}\right\}=\left\{q^{s}, q^{-s}\right\}$ is the Satake parameter attached to $\pi_{j, v}$. So we may take $n$ to be non-negative from here on. On the other hand, it is well known that $g\left(\chi_{v}, \psi_{q^{r}}\right)=0$ is $r<m$. Taking $r=m-n$ we get vanishing for $n>0$. Thus $n=0$, and we get (for any $s_{1}$ )

$$
P_{j, v}\left(f, \chi, s_{1}\right)=1
$$

On the other hand,

$$
L\left(s, \pi_{v} \otimes \chi_{v}\right)=1
$$

because $\pi_{v}$ is unramified, while $\chi_{v}$ is ramified. Note that for $v=p$, we have

$$
P_{j, p}\left(f, \chi, s_{1}\right)=f_{p}^{\wedge}\left(a_{p}\left(\varphi_{j}\right)\right) L\left(s_{1}+1 / 2, \pi_{j, p} \otimes \chi_{p}\right)
$$

Similarly, $Q_{j}\left(s_{2}\right)=L\left(s_{2}+1 / 2, \pi_{j}\right)$. The main assertion of the Proposition now follows on setting $s_{1}=s_{2}=0$.

\section{Proof of the Main Theorem}

The geometric side of the trace formula gives $I(f)$ as the sum of regular terms and two singular terms. By Corollary 1 , the contribution of the singular terms is $V_{N}^{-1} c_{k} L(1, \chi) S\left(f_{p}\right)$ where

$$
S\left(f_{p}\right)=F_{p}\left(n^{+}, f_{p}\right)+F_{p}\left(n^{-}, f_{p}\right)
$$

By Proposition 3.1, the regular terms are $O\left(N^{-k / 2+\varepsilon}\right)$ for all $\varepsilon>0$. In view of the expression for the spectral side in Section 4 , we obtain for all $\varepsilon>0$ :

$$
\sum \frac{L\left(\frac{1}{2}, \varphi_{j} \otimes \chi\right) L\left(\frac{1}{2}, \varphi_{j}\right)}{\left(\varphi_{j}, \varphi_{j}\right)} f^{\wedge}\left(a_{p}\left(\varphi_{j}\right)\right)=c_{k} L(1, \chi) S\left(f_{p}\right)+O\left(N^{-k / 2+\varepsilon}\right)
$$

The sum on the left of the formula above runs over newforms of level $N$ and oldforms which necessarily come from level 1 . Since $\chi(-1)=-1$, we see that the sign of the functional equation of $L(s, g \otimes \chi) L(s, g)$ is -1 for any form of level 1 , implying that $L(1 / 2, g \otimes \chi) L(1 / 2, g)$ is zero for any such $g$. There are two oldforms of level $N$ associated to $g$, which, in classical language, are $g(z)$ and $g_{N}(z):=g(N z)$. It is immediate that their Mellin transforms are related by $L\left(s, g_{N}\right)=N^{1-s} L(s, g)$. It follows that $L\left(1 / 2, g_{N} \otimes \chi\right) L\left(1 / 2, g_{N}\right)$ is also 0 . Therefore, we may restrict the sum to newforms.

In the next section, we prove that $S\left(f_{p}\right)=2 \mu_{p}\left(f_{p}^{\wedge}\right)$ where $\mu_{p}$ is the measure defined in the introduction. Since the map $f_{p} \rightarrow f_{p}^{\wedge}$ maps $\mathcal{H}_{p}$ onto the space 
of all polynomials on $[-2,2]$, it follows easily that we may replace $f_{p}^{\wedge}(x)$ by the characteristic function of a subinterval of $[-2,2]$ (cf. [Se]). This will complete the proof of Theorem A.

\section{The measure}

In this section, we write $f$ for $f_{p} \in \mathcal{H}_{p}$ and $\chi$ for $\chi_{p}$.

Proposition 6.1. $S(f)=2 \mu_{p}\left(f^{\wedge}\right)$

Let:

$$
\varphi(s)=\varphi_{f}(s)=\operatorname{Trace}\left(\pi_{s}(f)\right)
$$

where $\pi_{s}$ is the principal series representation unitarily induced from the character

$$
\left(\begin{array}{ll}
\alpha & 0 \\
0 & \beta
\end{array}\right) \rightarrow\left|\frac{\alpha}{\beta}\right|^{s}
$$

Then $\varphi(s)$ is a symmetric Laurent polynomial in $p^{s}$ and $p^{-s}$ and

$$
\varphi_{f}(s)=f^{\wedge}(x) \quad \text { where } \quad x=p^{s}+p^{-s}
$$

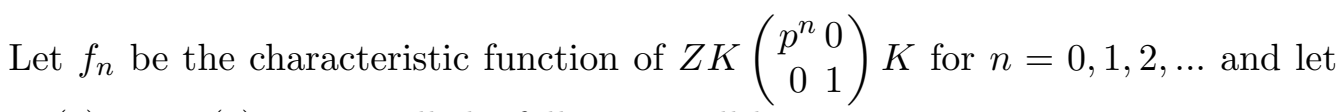
$\varphi_{n}(s)=\varphi_{f_{n}}(s)$. We recall the following well-known computation.

Lemma 9. $\varphi_{0}(s)=1$ and for $n \geq 1$,

$\left.\varphi_{n}(s)=p^{n / 2}\left[p^{n s}+p^{-n s}+\left(1-\frac{1}{p}\right)\left(p^{(n-2) s}+p^{(n-4) s}+\cdots+p^{-(n-4) s}\right)+p^{-(n-2) s}\right)\right]$

Proof. The coset $K\left(\begin{array}{cc}p^{n} & 0 \\ 0 & 1\end{array}\right) K$ is equal to a union of single cosets $g K$ where $g$ ranges over the following elements:

$$
\begin{array}{ll}
\left(\begin{array}{cc}
p^{n} & t \\
0 & 1
\end{array}\right) & t \in \mathcal{O} /\left(p^{n}\right) \\
\left(\begin{array}{cc}
p^{n-k} & t \\
0 & p^{k}
\end{array}\right) & t \in\left(\mathcal{O} / p^{n-k}\right)^{*} \quad k=1, \ldots, n
\end{array}
$$

Using this we easily calculate the action of $f_{n}$ on the principal series $\pi_{s}$ and we find the above formula.

We observe that since $f$ has compact support, the following integral is regular at $s_{2}=0$ :

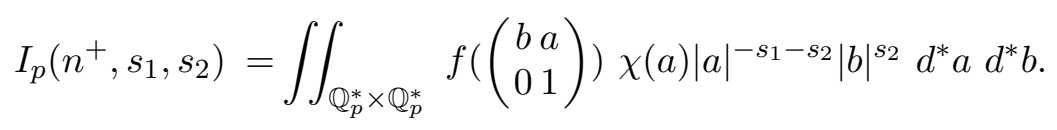


Thus we set $s_{2}=0$ and we define:

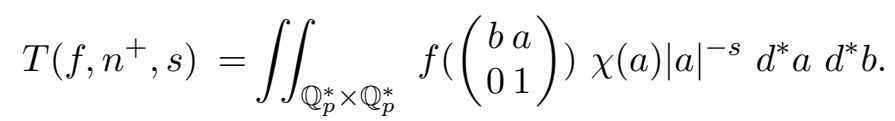

To evaluate $T\left(f_{n}\right)$ for $n \geq 0$, note that if

$$
\left(\begin{array}{ll}
b & a \\
0 & 1
\end{array}\right) \in Z K\left(\begin{array}{cc}
p^{n} & 0 \\
0 & 1
\end{array}\right) K
$$

then

$$
\left(\begin{array}{ll}
b & a \\
0 & 1
\end{array}\right) \in K\left(\begin{array}{cc}
p^{n+\alpha} & 0 \\
0 & p^{\alpha}
\end{array}\right) K
$$

for some $\alpha$. This is not possible unless $\alpha \leq 0$ and $b=p^{n+2 \alpha} u$ for some unit $u$. Furthermore, the gcd of $\left\{a, p^{n+2 \alpha}, 1\right\}$ is $p^{\alpha}$, so we must have $n+2 \alpha \geq \alpha$, that is, $\alpha \geq-n$. If $0>\alpha>-n$, then the gcd condition forces $a \in p^{\alpha} \mathbb{Z}_{p}^{*}$. If $\alpha=0$, then we just have $a \in \mathbb{Z}_{p}$ and if $\alpha=-n$, we have $\alpha \in p^{-n} \mathbb{Z}_{p}$.

Set $\delta=\chi(p)$. For $n=0$, we have:

$$
T\left(f_{0}, n^{+}, s\right)=\int_{u \in \mathbb{Z}_{p}^{*}} \int_{a \in \mathbb{Z}_{p}} \chi(a)|a|^{-s} d^{*} u d^{*} a=L_{p}(-s, \chi)
$$

For $n>0, T\left(f_{n}, n^{+}, s\right)$ is a sum of three terms:

$$
I=\int_{u \in \mathbb{Z}_{p}^{*}} \int_{a \in \mathbb{Z}_{p}} f\left(\left(\begin{array}{cc}
p^{n} u & a \\
0 & 1
\end{array}\right)\right) \chi(a)|a|^{-s} d^{*} u d^{*} a=L_{p}(-s, \chi)
$$

and

$$
\begin{aligned}
& I I=\int_{u \in \mathbb{Z}_{p}^{*}} \int_{a \in p^{-n} \mathbb{Z}_{p}} f\left(\left(\begin{array}{rr}
p^{-n} u a \\
0 & 1
\end{array}\right)\right) \chi(a)|a|^{-s} d^{*} u d^{*} a=\delta^{-n} p^{-n s} L_{p}(-s, \chi) \\
& I I I=\sum_{\alpha=1}^{n-1} \int_{u \in \mathbb{Z}_{p}^{*}} \int_{v \in \mathbb{Z}_{p}^{*}} f\left(\left(\begin{array}{c}
p^{n-2 \alpha} u p^{-\alpha} v \\
0 \\
1
\end{array}\right)\right) \chi\left(p^{-\alpha}\right)\left|p^{-\alpha}\right|^{-s} d^{*} u d^{*} v \\
& =\sum_{\alpha=1}^{n-1} \chi\left(p^{-\alpha}\right) p^{-\alpha s}=\frac{\delta^{-1} p^{-s}-\delta^{-n} p^{-n s}}{1-\delta^{-1} p^{-s}}=\frac{\delta^{1-n} p^{(1-n) s}-1}{1-\delta p^{s}} \\
& =L_{p}(-s, \chi)\left(\delta^{1-n} p^{(1-n) s}-1\right)
\end{aligned}
$$

We obtain:

$$
T\left(f_{n}, n^{+}, s\right)=L_{p}(-s, \chi)\left(\delta^{-n} p^{-n s}+\delta^{1-n} p^{(1-n) s}\right)
$$

and

$$
F_{p}\left(, n^{+}, f_{n}\right)=\lim _{s \rightarrow 0} \frac{T\left(f_{n}, n^{+}, s\right)}{L_{p}(-s, \chi)}=\delta^{-n}(1+\delta)
$$

We observe similarly that $I_{p}\left(n^{-}, s_{1}, s_{2}\right)$ is regular at $s_{1}=0$ : 


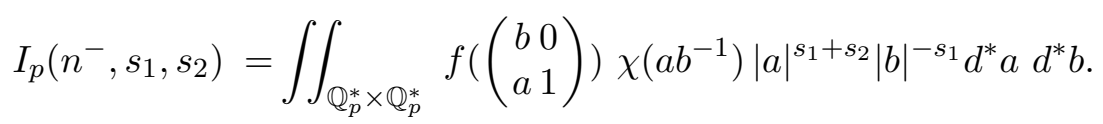

Thus we set $s_{1}=0$ and define

$$
T\left(f, n^{-}, s\right)=\iint_{\mathbb{Q}_{p}^{*} \times \mathbb{Q}_{p}^{*}} f\left(\left(\begin{array}{ll}
b & 0 \\
a & 1
\end{array}\right)\right) \chi\left(a b^{-1}\right)|a|^{s} d^{*} a d^{*} b .
$$

We evaluate $T\left(f_{n}, n^{-}, s\right)$ for $n \geq 0$. We first note that if

$$
\left(\begin{array}{ll}
b & 0 \\
a & 1
\end{array}\right) \in Z K\left(\begin{array}{cc}
p^{n} & 0 \\
0 & 1
\end{array}\right) K
$$

then

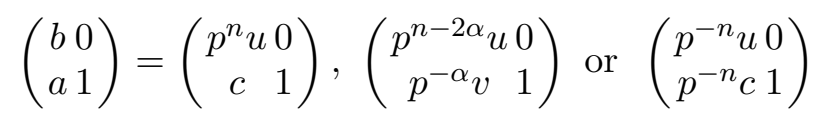

where $c \in \mathbb{Z}_{p}$ and $u, v$ are units in $\mathbb{Z}_{p}^{*}$, and $0 \leq \alpha \leq n$.

For $n=0$, we have:

$$
T\left(f_{0}, n^{-}, s\right)=\int_{u \in \mathbb{Z}_{p}^{*}} \int_{a \in \mathbb{Z}_{p}} \chi(a)|a|^{s} d^{*} u d^{*} a=L_{p}(s, \chi)
$$

For $n>0, T\left(f_{n}, n^{-}, s\right)$ is a sum of three terms:

$$
\begin{gathered}
I=\int_{u \in \mathbb{Z}_{p}^{*}} \int_{c \in \mathbb{Z}_{p}} \chi\left(p^{-n} c\right)|c|^{s} d^{*} u d^{*} c=\delta^{-n} L(s, \chi) \\
I I=\sum_{\alpha=1}^{n-1} \int_{u \in \mathbb{Z}_{p}^{*}} \int_{v \in \mathbb{Z}_{p}^{*}} \chi\left(p^{\alpha-n}\right)\left|p^{-\alpha}\right|^{s} d^{*} u d^{*} v \\
=\delta^{-n} \sum_{\alpha=1}^{n-1} \delta^{\alpha} p^{\alpha s}=\delta^{-n} \frac{\delta p^{s}-\delta^{n} p^{n s}}{1-\delta p^{s}}=\left(\delta^{1-n} p^{s}-p^{n s}\right) L(s, \chi) \\
I I I=\int_{u \in \mathbb{Z}_{p}^{*}} \int_{a \in \mathbb{Z}_{p}} \chi(a)\left|p^{-n} a\right|^{s} d^{*} u d^{*} a=p^{n s} L(s, \chi)
\end{gathered}
$$

We see that $T\left(f_{n}, n^{-}, s\right)=\delta^{-n}\left(1+\delta p^{s}\right) L(s, \chi)$ and that $F_{p}\left(, n^{-}, f_{n}\right)$ is equal to $F_{p}\left(, n^{+}, f_{n}\right)$ :

$$
F_{p}\left(, n^{-}, f_{n}\right)=\lim _{s \rightarrow 0} \frac{T\left(f_{n}, n^{-}, s\right)}{L_{p}(-s, \chi)}=\delta^{-n}(1+\delta)
$$

Now assume that $\delta=\chi(p)=-1$. Recall that in this case, $\mu_{p}=\mu_{-}$is the Plancherel measure on the Hecke algebra $\mathcal{H}_{p}$, which is defined by the property $\mu_{p}\left(f_{0}^{\wedge}\right)=1$ and $\mu_{p}\left(f_{n}^{\wedge}\right)=0$ for $n>0$. Our computations show that $S\left(f_{0}\right)=2$ 
and $S\left(f_{n}\right)=0$ for $n>0$ and thus $S(f)=2 \mu_{p}\left(f^{\wedge}\right)$. This proves Proposition 6.1 in the case $\delta=-1$.

To handle the case $\delta=-1$, we first treat $\delta$ as an indeterminate and find a function rational function $F(s)$ of $p^{s}$ such that the functional

$$
A_{\delta}(\varphi)=\frac{\log p}{2 \pi} \int_{-\frac{\pi}{\log p}}^{\frac{\pi}{\log p}} \varphi_{n}(i s) F(i s) d s
$$

satisfies

$$
A_{\delta}\left(\varphi_{n}\right)=\left\{\begin{array}{cr}
1 & n=0 \\
\delta^{n}+\delta^{-n} n \geq 1
\end{array}\right.
$$

For $\delta=1$, we have $A_{1}\left(\varphi_{n}\right)=F_{p}\left(, n^{+}, f_{n}\right)=F_{p}\left(, n^{-}, f_{n}\right)$.

\section{Proposition 6.2.}

$$
F(s)=1+\sum_{k=1}^{\infty} C_{k} p^{k\left(s-\frac{1}{2}\right)}
$$

where

$$
C_{k}=\delta^{k}+\delta^{-k}-(p-1)\left(\frac{\delta^{k-1}-\delta^{-k+1}}{\delta-\delta^{-1}}\right)
$$

Proof. We have

$$
C_{k}=\delta^{k}+\delta^{-k}-(p-1)\left(\delta^{k-2}+\delta^{k-4}+\cdots+\delta^{-k+4}+\delta^{-k+2}\right)
$$

Observe that

$$
\frac{\log p}{2 \pi} \int_{-\frac{\pi}{\log p}}^{\frac{\pi}{\log p}} p^{n s} p^{m s} d s=\left\{\begin{array}{l}
1 n+m=0 \\
0 \text { otherwise }
\end{array}\right.
$$

Therefore, $\int \varphi_{n}(s) F(s) d s$ is equal to

$$
C_{n}+\left(1-\frac{1}{p}\right) \sum_{j=1}^{N} p^{j} C_{n-2 j}=\delta^{n}+\delta^{-n}+\sum_{j=1}^{N} a_{j}\left(\delta^{n-2 j}+\delta^{-n+2 j}\right)
$$

where $N=\left[\frac{k}{2}\right]$. To verify the proposition, we must check that the coefficients $a_{j}$ are zero:

$$
\begin{aligned}
a_{j} & =-(p-1)-\left(1-\frac{1}{p}\right)\left[p(p-1)+p^{2}(p-1)+\cdots+p^{j}(p-1)-p^{j}\right] \\
& =1-p-\left(1-\frac{1}{p}\right)(-p)=0
\end{aligned}
$$


To write $F(s)$ as a rational function, we sum the geometric series. Let $T=$ $p^{s-\frac{1}{2}}$. Then:

$$
\begin{aligned}
F(s) & =1+\sum_{n \geq 1}\left[(\delta T)^{n}+\left(\delta^{-1} T\right)^{n}-\frac{(p-1)}{\delta-\delta^{-1}}\left(\delta^{-1}(\delta T)^{n}+\delta\left(\delta^{-1} T\right)^{n}\right)\right] \\
& =1+\sum_{n \geq 1} \frac{(\delta T)^{n}}{\delta-\delta^{-1}}\left[\delta-\delta^{-1}-p \delta^{-1}+\delta^{-1}\right]+\sum_{n \geq 1} \frac{\left.\delta^{-1} T\right)^{n}}{\delta-\delta^{-1}}\left[\delta-\delta^{-1}+p \delta-\delta\right] \\
& =1+\frac{\delta-p \delta^{-1}}{\delta-\delta^{-1}} \sum_{n \geq 1}(\delta T)^{n}-\frac{\delta^{-1}-p \delta}{\delta-\delta^{-1}} \sum_{n \geq 1}\left(\delta^{-1} T\right)^{n} \\
& =1+\frac{\delta-p \delta^{-1}}{\delta-\delta^{-1}} \frac{\delta T}{1-\delta T}-\frac{\delta^{-1}-p \delta}{\delta-\delta^{-1}} \frac{\delta^{-1} T}{1-\delta^{-1} T}
\end{aligned}
$$

Using Mathematica, we find the further simplification:

$$
F(s)=\frac{1-p T^{2}}{(T-\delta)\left(T-\delta^{-1}\right)}=\frac{1-p^{2 s}}{\left(1-\delta^{-1} p^{s-\frac{1}{2}}\right)\left(1-\delta p^{s-\frac{1}{2}}\right)}
$$

Now set $\delta=1$. Furthermore, since the Satake transforms are invariant under $s \rightarrow-s$, we may replace $F(s)$ by

$$
g(s)=\frac{1}{2}(F(s)+F(-s))=\frac{1}{2} \frac{\left(1-\frac{1}{p}\right)\left(2-p^{2 s}-p^{-2 s}\right)}{\left(1+\frac{1}{p}-p^{-\frac{1}{2}+s}-p^{-\frac{1}{2}-s}\right)^{2}}
$$

It follows that $S(f)=2 S_{0}\left(\varphi_{f}\right)$ where

$$
S_{0}(f)=\frac{\log p}{\pi} \int_{0}^{\frac{\pi}{\log p}} \varphi_{f}(i s) g(i s) d s
$$

Here is a graph of $g(i s)$ for the case $p=2$ and $\delta$ trivial.

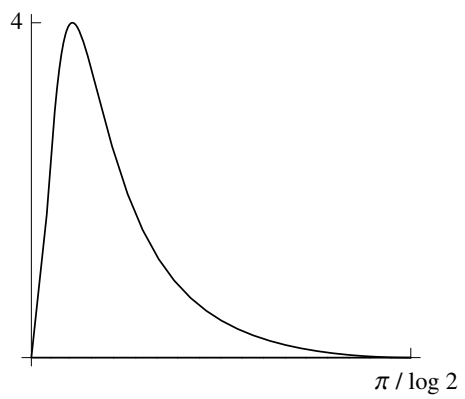


Now we make a change of variables $x=p^{i s}+p^{-i s}=2 \cos (s \log p)$. Then $x^{2}=2+p^{2 s}+p^{-2 s}$ and

$$
g(s)=\frac{1}{2} \frac{\left(1-\frac{1}{p}\right)\left(4-x^{2}\right)}{\left(1+\frac{1}{p}-p^{-\frac{1}{2}} x\right)^{2}}
$$

Furthermore,

$$
\begin{gathered}
d x=-2 \log (p) \sin (s \log p) d s=-\log (p) \sqrt{4-x^{2}} d s \\
\frac{\log (p)}{\pi} g(i s) d s=-\left(\frac{\log (p)}{\pi}\right) \frac{1}{2 \log (p)} \frac{\left(1-\frac{1}{p}\right) \sqrt{4-x^{2}}}{\left(1+\frac{1}{p}-p^{-\frac{1}{2}} x\right)^{2}} d x \\
=-\frac{1}{2 \pi} \frac{\left(1-\frac{1}{p}\right) \sqrt{4-x^{2}}}{\left(1+\frac{1}{p}-p^{-\frac{1}{2}} x\right)^{2}} d x
\end{gathered}
$$

and $S_{0}(f)=\int_{-2}^{2} f^{\wedge}(x) d u_{+}(x)$. This proves that $S(f)=2 \mu_{+}\left(f^{\wedge}\right)$, thus completing the proof of Proposition 6.1.

\section{Bibliography}

[A-S]: M. Abramowitz and I.A. Stegun, Handbook of mathematical functions, Dover edition (1965).

[Du]: W. Duke, The critical order of vanishing of automorphic $L$-functions with large level, Inventiones Math. 119, 165-174 (1995).

[GJ]: S. Gelbart and H. Jacquet, A relation between automorphic representations of GL(2) and GL(3), Ann. Scient. Éc. Norm. Sup. (4) 11 (1979), $471-542$.

[Gu]: J. Guo, On the positivity of the central critical values for automorphic $L$-functions for GL(2), Duke Mathematical Journal 83, No. 1, 157-190 (1996).

[IwS]: H. Iwaniec and P. Sarnak, The non-vanishing of central values of automorphic $L$-functions and Landau-Siegel zeros, Israel Journal of Math 120, 155-177 (2000).

[J]: H. Jacquet, Sur une résultat de Waldspurger, Ann. Scient. Éc. Norm. Sup., $4^{e}$ série, t. 19, 185-229 (1986).

[KMV]: E. Kowalski, P. Michel, J. VanderKam, Non-vanishing of high derivatives of automorphic $L$-functions at the center of the critical strip, J. Reine Angew. Math. 526 1-34 (2000). 
[Lu]: W. Luo, On the non-vanishing of Rankin-Selberg $L$-functions, Duke Math Journal 69, 411-427 (1993).

[Ro]: E. Royer, Facteurs $\mathbb{Q}$-simples de $J_{0}(N)$ de grande dimension et de grand rang, Bull. Soc. Math. France 128, no. 2, 219-248 (2000).

[Sa]: P. Sarnak, Statistical properties of eigenvalues of the Hecke operators, in Analytic number theory and Diophantine problems, 321-331, Progr. Math. 70, Birkhäuser, Boston (1987).

[Se]: J.-P. Serre, Répartition asymptotique des valeurs propres de l'opérateur de Hecke $T_{p}$, Journal of the AMS 10, no. 1, 75-102 (1997).

Dinakar Ramakrishnan

Professor of Mathematics

253-37 Caltech, Pasadena

CA 91125, USA

E-mail: dinakar@its.caltech.edu

Jonathan Rogawski

Professor of Mathematics

Department of Mathematics

University of California, Los Angeles

CA 90095, USA

E-mail: jonr@math.ucla.edu 